\title{
On the spectrum of two quantum layers coupled by a window
}

\author{
D. Borisov \\ Nuclear Physics Institute, Academy of Sciences, $25068 \check{R} e \check{z}$ \\ near Prague, Czechia \\ Bashkir State Pedagogical University, October Revolution St. 3a, \\ 450000 Ufa, Russia \\ E-mail: borisovdi@yandex.ru
}

\begin{abstract}
We consider the Dirichlet Laplacian in a domain two three-dimensional parallel layers having common boundary and coupled by a window. The window produces the bound states below the essential spectrum; we obtain two-sided estimates for them. It is also shown that the eigenvalues emerge from the threshold of essential spectrum as the window passes through certain critical shapes. We prove the necessary condition for the window to be of critical shape. Under an additional assumption we show that this condition is sufficient and obtain the asymptotic expansion for the emerging eigenvalue as well as for the associated eigenfunction.
\end{abstract}

\section{Introduction}

There is a number of works studying of the spectral properties the Dirichlet Laplacian in the unbounded domains like infinite planar strips or three-dimensional layers with some perturbations. The interest is stimulated by the applications of such models in quantum mechanics, in particular, in the theory of quantum waveguides. In the case the perturbation is absent, the system is trivial due to natural separation of variables, while the presence usually leads to various phenomena interesting both from physical and mathematical point of view.

One of the possible system attracting much attention is two adjacent parallel strips or layers coupled by the window(s) being bounded domain(s) cut out in the common boundary. The two-dimensional case was studied quite intensively, we refer here to [1], [2], [3], 4], [5], 6], [9] (see also references therein). It was shown that the perturbation by the window(s) is a negative one, i.e., it leads to the presence of the isolated bound states below the essential spectrum; the latter is invariant w.r.t. to the window(s). In the case of one window it was shown in [1], 3], 5] that widening the window one produces more and more isolated 
eigenvalues. They appear when the window's length passes through certain critical values; this phenomenon was studied in details and the asymptotics expansions for the emerging eigenvalues were obtained, see [1], [3], [9].

In the three-dimensional case corresponding to window-coupled layers P. Exner and S. Vugalter showed that a small window generates one simple isolated eigenvalue emerging from the threshold of the essential spectrum [6]. They also obtained two-sided asymptotic estimates for the eigenvalue. The asymptotics expansion for this eigenvalue has been constructed formally in [13]. In the present paper we treat the same system but for a finite window. The presence of a window leads to nonempty discrete spectrum; we obtain two-sided estimates for the eigenvalues. We show that enlargement of the window produces new isolated eigenvalues emerging from the continuum, and it happens in the way similar to the two-dimensional case. Namely, there are critical shapes of the window so that enlarging the latter one generates a new eigenvalue below the threshold no matter how the increment is small. We show that the necessary condition for such eigenvalue to emerge is the presence of non-trivial bounded resonance solution corresponding to the threshold of the essential spectrum. We describe all possible resonance solutions. We also prove that the presence of the bounded non-trivial resonance solution of certain type is sufficient to generate an eigenvalue below the essential spectrum. We also give the leading terms of the asymptotics expansions for this eigenvalue and the associated eigenfunction.

\section{Formulation of the problem and the main re- sults}

Let $x^{\prime}=\left(x_{1}, x_{2}\right), x=\left(x^{\prime}, x_{3}\right)$ be Cartesian coordinates in $\mathbb{R}^{2}$ and $\mathbb{R}^{3}$, respectively, and $\omega \subset \mathbb{R}^{2}$ be a bounded simply-connected domain having infinitely differentiable smooth boundary. We denote $\Pi_{\omega}:=\left\{x: x_{3} \in(-d, 0) \cup(0, \pi)\right\} \cup \omega, d \leqslant \pi$. In what follows the set $\omega \times\{0\}$ is referred to as window (cf. Figure).

The main object of our study is the Dirichlet Laplacian in $\Pi_{\omega}$ introduced rigorously as associated with the sesquilinear form

$$
\mathfrak{h}_{\omega}[u, v]:=(\nabla u, \nabla v)_{L_{2}\left(\Pi_{\omega}\right)}
$$

on $\stackrel{0}{W}{ }_{2}^{1}\left(\Pi_{\omega}\right)$, and we indicate it as $\mathcal{H}_{\omega}$. Hereinafter by $\stackrel{0}{W_{2}^{j}}\left(\Pi_{\omega}\right)$ we denote the subset of the functions in $W_{2}^{j}\left(\Pi_{\omega}\right)$ vanishing on $\partial \Pi_{\omega}$. Our main aim is to study the spectrum of $\mathcal{H}_{\omega}$.

In order to present the main results we require additional notations. Assuming $\omega \neq \emptyset$, in a small neighbourhood of $\partial \omega$ we introduce coordinates $(\tau, s)$, where $s$ is the arc length of $\partial \omega$, and $\tau$ is the distance to a point measured in the direction of the outward normal to $\partial \omega$. By $(r, \theta)$ we denote the polar coordinates corresponding to $\left(\tau, x_{3}\right)$. 


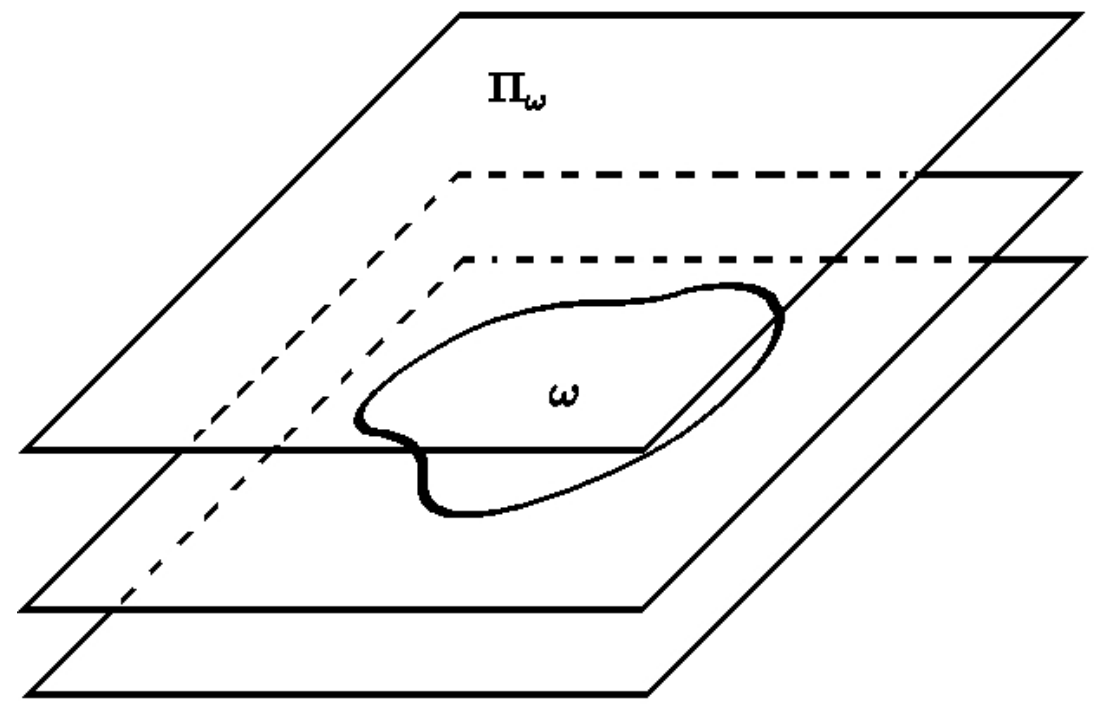

Figure. Window-coupled layers

Let $\chi=\chi(t) \in C^{\infty}(\mathbb{R})$ be a cut-off function vanishing as $t>1 / 3$ and equalling one as $t<1 / 4$. Given $\delta>0$ small enough, by $\mathfrak{V}_{\delta}$ we denote the set of the functions

$$
\begin{gathered}
u(x)=\left(a_{0}+\sum_{j=1}^{\infty} \mathrm{e}^{-\frac{2 \pi j r}{s_{0}}}\left(\frac{a_{j}}{\sqrt{j}} \cos \frac{2 \pi j s}{s_{0}}+\frac{\widetilde{a}_{j}}{\sqrt{j}} \sin \frac{2 \pi j s}{s_{0}}\right)\right) \sqrt{r} \sin \frac{\theta}{2}, \\
a_{j}, \widetilde{a}_{j} \in \mathbb{C}, \quad\|u\|_{\mathfrak{V}_{\delta}}^{2}:=\left|a_{0}\right|^{2}+\sum_{j=1}^{\infty}\left(\left|a_{j}\right|^{2}+\left|\widetilde{a}_{j}\right|^{2}\right)<\infty,
\end{gathered}
$$

defined on $T_{\delta}:=\{x: r<\delta\}$. Here $s_{0}$ is the length of $\partial \omega$. We will show that these functions are well-defined (see Theorem 1.11).

Given $S \subseteq \Pi_{\omega}$ and small $\delta>0$, by $\mathfrak{W}(\delta, S)$ we denote the set of the functions

$$
u(x)=u^{(0)}(x) \chi\left(\frac{r}{\delta}\right)+u^{(1)}(x),
$$

where $u^{(0)} \in \mathfrak{V}_{\delta}, u^{(1)} \in W_{2}^{2}(S), u=0$ on $\partial \prod_{\omega} \cap S$. We will employ the symbol $\mathcal{D}(\cdot)$ to indicate the domain of an operator.

Theorem 1.1. Suppose $\omega \neq \emptyset$. Then there exists $\delta_{0}>0$ such that $\mathcal{D}\left(\mathcal{H}_{\omega}\right)=$ $\mathfrak{W}\left(\delta, \Pi_{\omega}\right)$, and for each $u \in \mathcal{D}\left(\mathcal{H}_{\omega}\right)$

$$
\mathcal{H}_{\omega} u=-2 \nabla u^{(0)} \cdot \nabla \chi-u^{(0)} \Delta \chi-\Delta u^{(1)}, \quad \chi=\chi\left(\frac{r}{\delta_{0}}\right)
$$

The estimates

$$
C_{1}\left\|\mathcal{H}_{\omega} u\right\|_{L_{2}\left(\Pi_{\omega}\right)} \leqslant\left\|u^{(0)}\right\|_{\mathfrak{V}_{\delta}}+\left\|u^{(1)}\right\|_{W_{2}^{2}\left(\Pi_{\omega}\right)} \leqslant C_{2}\left\|\mathcal{H}_{\omega} u\right\|_{L_{2}\left(\Pi_{\omega}\right)}
$$




$$
\begin{aligned}
\left\|u^{(0)}\right\|_{W_{2}^{1}\left(T_{\delta}\right)} & +\left\|\frac{\partial}{\partial s} \nabla u^{(0)}\right\|_{L_{2}\left(T_{\delta}\right)}+\left\|r \frac{\partial^{2} u^{(0)}}{\partial \tau^{2}}\right\|_{L_{2}\left(T_{\delta}\right)} \\
& +\left\|r \frac{\partial^{2} u^{(0)}}{\partial \tau \partial x_{3}}\right\|_{L_{2}\left(T_{\delta}\right)}+\left\|r \frac{\partial^{2} u^{(0)}}{\partial x_{3}^{2}}\right\|_{L_{2}\left(T_{\delta}\right)} \leqslant C\left\|u^{(0)}\right\|_{\mathfrak{V}_{\delta}} .
\end{aligned}
$$

hold true, where the constants $C, C_{i}>0$ are independent of $u^{(0)}$ and $u^{(1)}$.

Let $\lambda_{i}=\lambda_{i}(\omega)$ be the isolated eigenvalues of $\mathcal{H}_{\omega}$ taken counting multiplicity and ordered in the non-decreasing order. By $\sigma(\cdot), \sigma_{\text {ess }}(\cdot), \sigma_{\text {disc }}(\cdot)$ we denote the spectrum, the essential spectrum and the discrete one of the operator. We will also use the symbol \#A to indicate the number of the elements in a set $A$.

Theorem 1.2. The essential spectrum of $\mathcal{H}_{\omega}$ coincides with $[1,+\infty)$. The discrete spectrum consists of a finite number of the eigenvalues satisfying inequalities

$$
\frac{\pi^{2}}{(\pi+d)^{2}}+\mu_{i}^{(N)} \leqslant \lambda_{i}(\omega) \leqslant \frac{\pi^{2}}{(\pi+d)^{2}}+\mu_{i}^{(D)}
$$

where $\mu_{i}^{(N)}, \mu_{i}^{(D)}$ are the eigenvalues of the Neumann and Dirichlet Laplacian in $\omega$, respectively. The number of the eigenvalues of $\mathcal{H}_{\omega}$ is estimated as

$$
\#\left\{\mu_{i}^{(D)}: \mu_{i}^{(D)}<\frac{2 \pi d+d^{2}}{(\pi+d)^{2}}\right\} \leqslant \# \sigma_{\text {disc }}\left(\mathcal{H}_{\omega}\right) \leqslant \#\left\{\mu_{i}^{(N)}: \mu_{i}^{(N)}<\frac{2 \pi d+d^{2}}{(\pi+d)^{2}}\right\} .
$$

We denote $B_{\rho}(c):=\left\{x^{\prime}:\left|x^{\prime}-c\right|<\rho\right\}$.

Theorem 1.3. Let $\omega=\omega(t) \subset \mathbb{R}^{2}$ be a family of bounded simply-connected domains having infinitely differentiable boundary and satisfying the assumption

(A1). For each $t_{0} \in(0,+\infty)$ and $t$ close to $t_{0}$ there exist diffeomorphism $\mathcal{M}\left(t_{0}, t\right) \in$ $C^{3}$ defined in the vicinity of $\omega\left(t_{0}\right)$ such that $\mathcal{M}\left(t_{0}, t\right) \omega\left(t_{0}\right)=\omega(t), \mathcal{M}_{0}\left(t_{0}, t_{0}\right)=$ I; the components of $\mathcal{M}\left(t_{0}, t\right)$ and their derivatives up to the second order are continuous jointly w.r.t. spatial variables and $t$.

Then the eigenvalues of $\mathcal{H}_{\omega(t)}$ are continuous w.r.t. to $t$. If, in addition, the assumption

(A2). There exist $\rho_{i}=\rho_{i}(t), i=1,2$, such that $B_{\rho_{1}(t)}(0) \subset \omega(t) \subset B_{\rho_{2}(t)}(0)$, $t \in[0,+\infty)$, and

$$
\lim _{t \rightarrow+\infty} \rho_{1}(t)=+\infty, \quad \lim _{t \rightarrow+0} \rho_{2}(t)=0 ;
$$

$$
\omega\left(t_{1}\right) \subset \omega\left(t_{2}\right) \text { for all } t_{1}<t_{2} ;
$$

holds true, then there exists an infinite sequence $0=t_{1}<t_{2} \leqslant t_{3} \leqslant \ldots$, such that $\# \sigma_{\text {disc }}\left(\mathcal{H}_{\omega(t)}\right)=n, t \in\left(t_{n}, t_{n+1}\right], t_{n} \rightarrow+\infty, n \rightarrow+\infty$, and $\lambda_{n}(\omega(t)) \rightarrow 1-0$, $t \rightarrow t_{n}+0$. 
This theorem states that there exist critical shapes of $\omega$ such that enlarging them one produces new eigenvalue(s) below the essential spectrum. The next part of our results describes how such eigenvalues emerge. First we state

Lemma 1.4. The problem

$$
-\Delta \Psi=\Psi \quad \text { in } \quad \Pi_{\omega}, \quad \Psi=0 \quad \text { on } \quad \partial \Pi_{\omega},
$$

has at most finite number of bounded non-trivial solutions assumed to be even w.r.t. $x_{3}$ if $d=\pi$. They can be chosen so that there is at most one solution behaving at infinity as

$$
\Psi=\sin x_{3}+\mathcal{O}\left(\left|x^{\prime}\right|^{-2}\right), \quad x^{\prime} \rightarrow+\infty, \quad x_{3} \in(0, \pi)
$$

at most two solutions behaving as

$$
\Psi=\frac{c_{1} x_{1}+c_{2} x_{2}}{\left|x^{\prime}\right|^{2}} \sin x_{3}+\mathcal{O}\left(\left|x^{\prime}\right|^{-3}\right), \quad x^{\prime} \rightarrow+\infty, \quad x_{3} \in(0, \pi), \quad\left|c_{1}\right|^{2}+\left|c_{2}\right|^{2}=1
$$

and a finite number of solutions belonging to $L_{2}\left(\Pi_{\omega}\right)$. Each of these solutions is infinitely differentiable up to the boundary except $\partial \omega \times\{0\}$, while in the vicinity of $\partial \omega \times\{0\}$ it behaves as

$$
\Psi(x)=l_{\Psi}(s) \sqrt{r} \sin \frac{\theta}{2}+\mathcal{O}(r), \quad r \rightarrow 0,
$$

where $l_{\Psi} \in C^{\infty}(\partial \omega)$.

Given $\omega$, we consider the family of bounded domains $\omega_{\varepsilon} \subset \mathbb{R}^{2}$ whose boundaries are $\partial \omega_{\varepsilon}:=\left\{x^{\prime}: \tau=\varepsilon \beta(s)\right\}$, where $\varepsilon \rightarrow+0$, and $\beta \in C^{\infty}(\partial \omega)$ is an arbitrary function.

Theorem 1.5. Suppose the problem (1.7) has no bounded non-trivial solution assumed to be even w.r.t. $x_{3}$ if $d=\pi$. Then the operator $\mathcal{H}_{\omega_{\varepsilon}}$ has no eigenvalues converging to one as $\varepsilon \rightarrow+0$.

We introduce two-valued symbol, $\gamma:=1$, if $d<\pi$, and $\gamma:=2$, if $d=\pi$.

Theorem 1.6. Suppose the problem (1.7) has the unique bounded solution $\Psi$ assumed to be even w.r.t. $x_{3}$ if $d=\pi$, and it satisfies (1.8). Then $l_{\Psi} \not \equiv 0$, and there exists the unique solution $\widetilde{\Psi}$ to (1.7) satisfying the conditions

$$
\begin{aligned}
& \widetilde{\Psi}(x)=\frac{l_{\Psi}(s) \beta(s)}{2 \sqrt{r}} \sin \frac{\theta}{2}+l_{\widetilde{\Psi}}(s) \sqrt{r} \sin \frac{\theta}{2}+\mathcal{O}(r), \quad r \rightarrow 0, \\
& \widetilde{\Psi}(x)=c \ln \left|x^{\prime}\right| \sin x_{3}+\mathcal{O}\left(\left|x^{\prime}\right|^{-1}\right), \quad\left|x^{\prime}\right| \rightarrow+\infty, \quad x_{3} \in(0, \pi),
\end{aligned}
$$

where $\widetilde{l}_{\Psi} \in C^{\infty}(\partial \omega)$. If

$$
\mathfrak{i}_{1}:=\frac{1}{2 \gamma} \int_{\partial \omega} \beta l_{\Psi}^{2} \mathrm{~d} s>0 ; \quad \text { or } \quad \mathfrak{i}_{1}=0, \quad \mathfrak{i}_{2}:=\frac{1}{2 \gamma} \int_{\partial \omega} \beta l_{\Psi} l_{\widetilde{\Psi}} \mathrm{d} s>0,
$$


then there exists the unique eigenvalue of $\mathcal{H}_{\omega_{\varepsilon}}$ converging to $1-0$ as $\varepsilon \rightarrow+0$; it is simple, and

$$
\begin{array}{ll}
\lambda_{\varepsilon}=1-4 \mathrm{e}^{-2 \mathrm{C}+2 \frac{\mathfrak{i}_{2}}{\mathfrak{i}_{1}^{2}}} \mathrm{e}^{-\frac{2}{\varepsilon i_{1}}}(1+\mathcal{O}(\varepsilon)), & \text { if } \mathfrak{i}_{1}>0, \\
\lambda_{\varepsilon}=1-\mathrm{e}^{-\frac{2}{\varepsilon^{2} \mathrm{i}_{2}}}(c+\mathcal{O}(\varepsilon)), & \text { if } \mathfrak{i}_{1}=0, \quad \mathfrak{i}_{2}>0,
\end{array}
$$

where $c$ is a constant, $\mathrm{C}$ is the Euler constant. The associated eigenfunction satisfies the identity

$$
\psi_{\varepsilon}=\Psi+\mathcal{O}(\sqrt{\varepsilon})
$$

in the norms of $W_{2}^{1}(S)$ and $W_{2}^{2}\left(S \backslash T_{\delta}\right)$ for each bounded fixed domain $S \subset \Pi_{\omega_{\varepsilon}}$ and $\delta>0$. It decays exponentially at infinity,

$$
\psi_{\varepsilon}=\mathcal{O}\left(\varepsilon^{-\sqrt{1-\lambda_{\varepsilon}}\left|x^{\prime}\right|}\left|x^{\prime}\right|^{-1}\right), \quad\left|x^{\prime}\right| \rightarrow+\infty .
$$

If

$$
\mathfrak{i}_{1}<0 ; \quad \text { or } \quad \mathfrak{i}_{1}=0, \quad \mathfrak{i}_{2}<0,
$$

then the operator $\mathcal{H}_{\omega_{\varepsilon}}$ has no eigenvalues converging to $1-0$ as $\varepsilon \rightarrow+0$.

We observe that the leading terms in the asymptotics (1.13) are discontinuous as $d \rightarrow \pi$; this is due to the presence of $\gamma$ in the formulas. The similar phenomenon was found formally in [13] in the case of small window. We note that it occurs in two-dimensional case as well, see [1].

Theorem [1.5 states that the necessary condition for the eigenvalues to emerge is the presence of a bounded non-trivial solution to (1.7). There is a number of cases corresponding to various non-trivial solutions. One of the possible cases treats theorem 1.6, other cases remain open. It is an interesting question to obtain the results similar to Theorem 1.6 for the remaining cases. In particular, we conjecture that the total multiplicity of the emerging eigenvalues coincides with the number of bounded non-trivial solutions to (1.7). The other conjecture is that if there exists the unique bounded non-trivial solution to (1.7), then the eigenvalue emerges if $\mathfrak{i}_{1}>0$, and does not if $\mathfrak{i}_{1}<0$. Moreover, if the eigenvalue emerges, its asymptotics should depend on the behaviour at infinity of the non-trivial solution. Namely, we conjecture that

$$
\lambda_{\varepsilon}=1-c \frac{\varepsilon}{|\ln \varepsilon|}+\ldots,
$$

if the non-trivial solution satisfies (1.9), and

$$
\lambda_{\varepsilon}=1-c \varepsilon+\ldots,
$$

if the non-trivial solution belongs to $L_{2}(\Pi)$, where $c$ are some constants. One of the motivations to these asymptotics is the results of [12] where the two-dimensional Schrödinger operator on the plane perturbed by a fast decaying potential was considered. They addressed the same question on describing the behaviour of the eigenvalues emerging from the threshold of the essential spectrum. The asymptotics similar to (1.13) were shown to occur in some cases, while in the other cases the asymptotics similar to (1.16), (1.17) were valid. 


\section{Domain of $\mathcal{H}_{\omega}$}

The section is devoted to the proof of Theorem 1.1. We begin with the series of auxiliary lemmas and notations. We denote $\Omega_{\delta}:=\left\{\left(\tau, x_{3}\right): r<\delta\right\} \backslash\left\{\left(\tau, x_{3}\right): x_{3}=\right.$ $0, \tau>0\}$, where $\delta>0$ is small enough.

Lemma 2.1. For each $g \in L_{2}\left(\Omega_{\delta}\right)$ there exists the unique generalized solution $v \in \stackrel{0}{W}_{2}^{1}(\Omega)$ to

$$
\Delta_{\tau, x_{3}} v=g \quad \text { in } \quad \Omega, \quad v=0 \quad \text { on } \quad \partial \Omega .
$$

It can be represented as $v=v^{(0)}+v^{(1)}, v^{(0)}=\alpha \sqrt{r} \sin \frac{\theta}{2}$, where $v^{(1)} \in \stackrel{0}{W}_{2}^{2}(\Omega)$. The estimate

$$
|\alpha|+\left\|v^{(1)}\right\|_{W_{2}^{2}\left(\Omega_{\delta}\right)} \leqslant C\|g\|_{L_{2}\left(\Omega_{\delta}\right)}
$$

holds true, where the constant $C$ is independent of $g$ and $\delta$.

Proof. It is sufficient to give the proof for two subcases corresponding to the function $g$ being odd or even w.r.t. $x_{3}$. In both cases the unique solvability of (2.1) follows from the standard results in theory of generalized solutions to elliptic boundary value problems.

If $g$ is odd, the generalized solution to (2.1) is odd w.r.t. $x_{3}$ and hence $v=0$ as $x_{3}=0$. Thus, this function solves the boundary value problem like (2.1) but in the half-disk $\Omega_{\delta} \cap\left\{\left(\tau, x_{3}\right): x_{3}>0\right\}$. By the smoothness improving theorems we thus obtain that $v \in W_{2}^{2}\left(\Omega_{\delta}\right), \alpha=0$, and the estimate (2.2) is valid.

Suppose now that $g$ is even w.r.t. $x_{3}$. We expand $g$ into the Fourier series

$$
g\left(\tau, x_{3}\right)=\sum_{j=0}^{\infty} g_{2 j+1}(r) \sin \frac{2 j+1}{2} \theta, \quad g_{p}(r)=\frac{1}{\pi} \int_{0}^{2 \pi} g\left(\tau, x_{3}\right) \sin \frac{p \theta}{2} \mathrm{~d} \theta,
$$

which holds true in $L_{2}\left(\Omega_{\delta}\right)$-norm. This fact can be established by analogy with the proof of Lemma 3.2 in [1]. The Parseval identity

$$
\|g\|_{L_{2}(\Omega)}^{2}=\pi \sum_{j=0}^{\infty} \int_{0}^{\delta}\left|g_{2 j+1}(r)\right|^{2} r \mathrm{~d} r
$$

is valid. We now solve (2.1) by separation of variables,

$$
\begin{gathered}
v\left(\tau, x_{3}\right)=\sum_{j=0}^{\infty} v_{2 j+1}(r) \sin \frac{2 j+1}{2} \theta \\
v_{p}(r):=\frac{r^{\frac{p}{2}}}{p} \int_{\delta}^{r} t^{-\frac{p}{2}+1} g_{p}(t) \mathrm{d} t-\frac{r^{-\frac{p}{2}}}{p} \int_{0}^{r} t^{\frac{p}{2}+1} g_{p}(t) \mathrm{d} t+\frac{r^{\frac{p}{2}} \delta^{-p}}{p} \int_{0}^{\delta} t^{\frac{p}{2}+1} g_{p}(t) \mathrm{d} t .
\end{gathered}
$$


Let us first analyse the first term in this series. We define

$$
\begin{gathered}
\widetilde{v}_{1}(r):=v_{1}(r)-\alpha \sqrt{r}=r^{\frac{1}{2}} \int_{0}^{r} t^{\frac{1}{2}} g_{1}(t) \mathrm{d} t-r^{-\frac{1}{2}} \int_{0}^{r} t^{\frac{3}{2}} g_{1}(t) \mathrm{d} t, \\
\alpha:=\frac{1}{\pi} \int_{\Omega_{\delta}} r^{-\frac{1}{2}}\left(\frac{r}{\delta}-1\right) g \sin \frac{\theta}{2} \mathrm{~d} \tau \mathrm{d} x_{3} .
\end{gathered}
$$

It is easy to estimate the constant $\alpha$ :

$$
|\alpha|^{2} \leqslant \frac{\|g\|_{L_{2}\left(\Omega_{\delta}\right)^{2}}}{\pi^{2}} \int_{\Omega_{\delta}} r^{-1}\left(\frac{r}{\delta}-1\right)^{2} \mathrm{~d} \tau \mathrm{d} x_{3}=\frac{2 \delta}{3 \pi}\|g\|_{L_{2}\left(\Omega_{\delta}\right)^{2} .}
$$

Employing the estimate

$$
\int_{0}^{r} t^{\frac{3}{2}}\left|g_{1}(t)\right| \mathrm{d} t \leqslant r \int_{0}^{r} t^{\frac{1}{2}}\left|g_{1}(t)\right| \mathrm{d} t
$$

we check that

$$
\begin{aligned}
\left\|\widetilde{v}_{1}(r) \sin \frac{\theta}{2}\right\|_{W_{2}^{2}\left(\Omega_{\delta}\right)}^{2} \leqslant C \int_{0}^{\delta}\left(\left|\widetilde{v}_{1}^{\prime \prime}\right|^{2} r+\left|\widetilde{v}_{1}^{\prime}\right|^{2} r^{-1}+\left|\widetilde{v}_{1}\right|^{2} r^{-3}\right) \mathrm{d} r \\
\leqslant C \int_{0}^{\delta} r^{-2}\left(\int_{0}^{r} t^{\frac{1}{2}}\left|g_{1}(t)\right|^{2} \mathrm{~d} t\right)^{2} \mathrm{~d} r \\
\leqslant C \int_{0}^{\delta} r^{-\frac{3}{2}} \int_{0}^{r} t^{\frac{3}{2}}\left|g_{1}(t)\right|^{2} \mathrm{~d} t \mathrm{~d} r \leqslant C \int_{0}^{\delta} r\left|g_{1}(r)\right|^{2} \mathrm{~d} r \leqslant C\|g\|_{L_{2}\left(\Omega_{\delta}\right)}^{2},
\end{aligned}
$$

where the constant $C$ is independent of $g$ and $\delta$. In view of the inequality obtained and (2.5) it remains to show that the series $\sum_{j=1}^{\infty} v_{2 j+1}(r) \sin \frac{(2 j+1) \theta}{2}$ converges in $W_{2}^{2}\left(\Omega_{\delta}\right)$-norm and to estimate its norm by $\|g\|_{L_{2}\left(\Omega_{\delta}\right)}$. Hence, we should show that

$$
\sum_{j=1}^{\infty}\left\|v_{2 j+1}(r) \sin \frac{(2 j+1) \theta}{2}\right\|_{W_{2}^{2}\left(\Omega_{\delta}\right)} \leqslant C\|g\|_{L_{2}\left(\Omega_{\delta}\right)}
$$

Employing the definition of $v_{p},(2.3)$, and the estimate

$$
\left|\int_{0}^{\delta} t^{\frac{p}{2}+1} g_{p}(t) \mathrm{d} t\right|^{2} \leqslant \frac{\delta^{p+2}}{p+2} \int_{0}^{\delta} r\left|g_{p}(r)\right|^{2} \mathrm{~d} r
$$


we see that it is sufficient to check that

$\sum_{j=1}^{\infty} j^{2} \int_{0}^{\delta}\left(r^{2 j-2}\left(\int_{r}^{\delta} t^{-j+\frac{1}{2}} g_{j}(t) \mathrm{d} t\right)^{2}+r^{-2 j-4}\left(\int_{0}^{r} t^{j+\frac{3}{2}} g_{j}(t) \mathrm{d} t\right)^{2}\right) \mathrm{d} r \leqslant C\|g\|_{L_{2}\left(\Omega_{\delta}\right)}^{2}$,

where the constant $C$ is independent of $g$ and $\delta$. This estimate follows from (2.3) and the chain of inequalities

$$
\begin{aligned}
\int_{0}^{\delta} r^{2 j-2} & \left(\int_{r}^{\delta} t^{-j+\frac{1}{2}} g_{j}(t) \mathrm{d} t\right)^{2} \mathrm{~d} r \leqslant \int_{0}^{\delta} \frac{2 r^{j+\frac{1}{2}}}{5-2 j}\left(1-\frac{r^{j-\frac{5}{2}}}{\delta^{j-\frac{5}{2}}}\right) \int_{r}^{\delta} t^{-j-\frac{1}{2}}\left|g_{j}(t)\right|^{2} \mathrm{~d} t \mathrm{~d} r \\
& \leqslant \int_{0}^{\delta} \frac{2 r^{j+\frac{1}{2}}}{|5-2 j|} \int_{r}^{\delta} t^{-j-\frac{1}{2}}\left|g_{j}(t)\right|^{2} \mathrm{~d} t \mathrm{~d} r \leqslant \frac{4}{|5-2 j|(2 j+3)} \int_{0}^{\delta} r\left|g_{j}(r)\right|^{2} \mathrm{~d} r \\
\int_{0}^{\delta} r^{-2 j-4} & \left(\int_{0}^{r} t^{j+\frac{3}{2}} g_{j}(t) \mathrm{d} t\right)^{2} \mathrm{~d} r \leqslant \int_{0}^{\delta} \frac{2 r^{-j-\frac{1}{2}}}{2 j+7} \int_{0}^{r} t^{j+\frac{1}{2}}\left|g_{j}(t)\right|^{2} \mathrm{~d} t \\
& \leqslant \frac{4}{(2 j+7)(2 j-1)} \int_{0}^{\delta} r\left|g_{j}(r)\right|^{2} \mathrm{~d} r,
\end{aligned}
$$

where we have integrated by parts.

Lemma 2.2. For each $f \in L_{2}\left(T_{\delta}\right)$ there exists the unique generalized solution $u \in \stackrel{0}{W_{2}^{1}}\left(T_{\delta}\right)$ to the problem

$$
\Delta_{\tau, x_{3}, s} u=f \quad \text { in } \quad T_{\delta}, \quad u=0 \quad \text { on } \quad \partial T_{\delta} .
$$

It can be represented as

$$
u=u^{(0)}+u^{(1)}, \quad u^{(0)} \in \mathfrak{V}_{\delta}, \quad u^{(1)} \in W_{2}^{2}\left(T_{\delta}\right) .
$$

The estimates (1.3) and

$$
\left\|u^{(0)}\right\|_{\mathfrak{V}_{\delta}}+\left\|u^{(1)}\right\|_{W_{2}^{2}\left(T_{\delta}\right)} \leqslant C\|f\|_{L_{2}\left(T_{\delta}\right)}
$$

are valid.

Proof. The unique solvability of (2.7) is obvious. We separate variables and obtain:

$$
\begin{aligned}
& f(x)=f_{0}\left(\tau, x_{3}\right)+\sum_{j=1}^{\infty}\left(f_{j}\left(\tau, x_{3}\right) \cos \frac{2 \pi j s}{s_{0}}+\tilde{f}_{j}\left(\tau, x_{3}\right) \sin \frac{2 \pi j s}{s_{0}}\right), \\
& f_{0}=\frac{1}{s_{0}} \int_{0}^{s_{0}} f \mathrm{~d} s, \quad f_{j}=\frac{2}{s_{0}} \int_{0}^{s_{0}} f \cos \frac{2 \pi j s}{s_{0}} \mathrm{~d} s, \quad \tilde{f}_{j}=\frac{2}{s_{0}} \int_{0}^{s_{0}} f \sin \frac{2 \pi j s}{s_{0}} \mathrm{~d} s,
\end{aligned}
$$




$$
u(x)=u_{0}\left(\tau, x_{3}\right)+\sum_{j=1}^{\infty}\left(u_{j}\left(\tau, x_{3}\right) \cos \frac{2 \pi j s}{s_{0}}+\widetilde{u}_{j}\left(\tau, x_{3}\right) \sin \frac{2 \pi j s}{s_{0}}\right),
$$

where the series for $f$ converges in $L_{2}\left(T_{\delta}\right)$, and the coefficients of (2.10) are the generalized solutions to

$$
\left(\Delta_{\tau, x_{3}}-N^{2}\right) v=g \quad \text { in } \quad \Omega_{\delta}, \quad v=0 \quad \text { on } \quad \partial \Omega_{\delta},
$$

where $N=2 \pi j / s_{0}$, and $g=f_{j}$ or $g=\widetilde{f}_{j}$. These problems are uniquely solvable in $\stackrel{0}{W_{2}^{1}}\left(\Omega_{\delta}\right)$. By [11, Ch. V, Sec. 3.5, Eq. (3.16)] and the identity

$$
\|\nabla v\|_{L_{2}\left(\Omega_{\delta}\right)}^{2}-N^{2}\|v\|_{L_{2}\left(\Omega_{\delta}\right)}^{2}=(g, v)_{L_{2}\left(\Omega_{\delta}\right)}
$$

we have the estimates

$$
\|v\|_{L_{2}\left(\Omega_{\delta}\right)} \leqslant \frac{C}{N^{2}+1}\|g\|_{L_{2}\left(\Omega_{\delta}\right)}, \quad\|v\|_{W_{2}^{1}\left(\Omega_{\delta}\right)} \leqslant \frac{C}{N+1}\|g\|_{L_{2}\left(\Omega_{\delta}\right)},
$$

where the constant $C$ is independent of $g, N$, and $\delta$. Thus, the series (2.10) converges in $W_{2}^{1}\left(\Omega_{\delta}\right)$-norm and therefore gives the generalized solution to (2.7). This solution solves also (2.1), where the right-hand side is $\left(g+N^{2} v\right)$. We take into account (2.11) and apply Lemma 2.1 to conclude that the function $v$ can be represented as $v=\alpha \sqrt{r} \sin \frac{\theta}{2}+v^{(1)}$, where $\alpha$ and $v^{(1)} \in W_{2}^{2}\left(\Omega_{\delta}\right)$ satisfy (2.2).

Let us estimate $\alpha$ more precisely. It follows from Lemma 2.1 that the first term in the series (2.4) for $v$ satisfies the relations

$$
v_{0}(r) \sin \frac{\theta}{2}=\alpha \sqrt{r} \sin \frac{\theta}{2}+\widetilde{v}_{0}\left(\tau, x_{3}\right), \quad v_{0}(r)=\frac{1}{\pi} \int_{0}^{2 \pi} v \sin \frac{\theta}{2} \mathrm{~d} \theta
$$

where $\widetilde{v}_{0} \in W_{2}^{2}\left(\Omega_{\delta}\right)$. The function $v_{0}$ solves the problem

$$
\left(\frac{d}{d r} r \frac{d}{d r}-\frac{1}{4 r^{2}}-N^{2}\right) v_{0}=g_{0} \quad \text { in } \quad(0, \delta), \quad v_{0}(\delta)=0, \quad g_{0}=\frac{1}{\pi} \int_{0}^{2 \pi} g \sin \frac{\theta}{2} \mathrm{~d} \theta .
$$

and obeys the condition $v_{0}(r) \sin \frac{\theta}{2} \in \stackrel{0}{W_{2}^{1}}\left(\Omega_{\delta}\right)$. Hence,

$$
v_{0}(r)=\int_{0}^{r} g_{0}(t) \frac{\sqrt{t} \sinh N(r-t)}{N \sqrt{r}} \mathrm{~d} t+\frac{\sinh N r}{N \sqrt{r} \sinh N \delta} \int_{0}^{\delta} g_{0}(t) \sqrt{t} \sinh N(t-\delta) \mathrm{d} t .
$$

Proceeding as in (2.6), we check that

$$
\frac{\sin \frac{\theta}{2}}{N \sqrt{r}} \int_{0}^{r} g_{0}(t) \sqrt{t} \sinh N(r-t) \mathrm{d} t \in W_{2}^{2}\left(\Omega_{\delta}\right)
$$


and therefore

$$
\begin{aligned}
& \alpha=\int_{0}^{\delta} \frac{\sqrt{t} \sinh N(t-\delta)}{\sinh N \delta} g_{0}(t) \mathrm{d} t \\
& |\alpha|^{2} \leqslant \int_{0}^{\delta} \frac{\sinh ^{2} N(\delta-t)}{\sinh ^{2} N \delta} \mathrm{d} t \int_{0}^{\delta} t\left|g_{0}(t)\right|^{2} \mathrm{~d} t \leqslant \frac{C}{N}\|g\|_{L_{2}\left(\Omega_{\delta}\right)},
\end{aligned}
$$

where $C=\max _{[0,+\infty)} \frac{\sinh 2 t-2 t}{4 \sinh ^{2} t}<\infty$. It is easy to check that for $N \neq 0$

$$
\left\|\sqrt{r} \mathrm{e}^{-N r} \sin \frac{\theta}{2}\right\|_{L_{2}\left(\Omega_{\delta}\right)}^{2}=\pi \int_{0}^{\delta} r^{2} \mathrm{e}^{-2 N r} \mathrm{~d} r \leqslant \frac{\pi}{N^{3}} \int_{0}^{+\infty} t^{2} \mathrm{e}^{-2 t} \mathrm{~d} t=\frac{\pi}{4 N^{3}} .
$$

In the same way one can make sure

$$
\left\|\sqrt{r} \mathrm{e}^{-N r} \sin \frac{\theta}{2}\right\|_{W_{2}^{1}\left(\Omega_{\delta}\right)}^{2} \leqslant \frac{C}{N}, \quad\left\|\sqrt{r}\left(\mathrm{e}^{-N r}-1\right) \sin \frac{\theta}{2}\right\|_{W_{2}^{2}\left(\Omega_{\delta}\right)}^{2} \leqslant C N
$$

where the constant $C$ is independent of $N$ and $\delta$. By (2.11) and (2.2) we conclude now that

$$
\begin{aligned}
& \left\|v-\alpha \sqrt{r} \mathrm{e}^{-N r} \sin \frac{\theta}{2}\right\|_{L_{2}\left(\Omega_{\delta}\right)} \leqslant \frac{C}{N^{2}+1}\|g\|_{L_{2}\left(\Omega_{\delta}\right)}, \\
& \left\|v-\alpha \sqrt{r} \mathrm{e}^{-N r} \sin \frac{\theta}{2}\right\|_{W_{2}^{1}\left(\Omega_{\delta}\right)} \leqslant \frac{C}{N+1}\|g\|_{L_{2}\left(\Omega_{\delta}\right)}, \\
& \left\|v^{(1)}-\alpha \sqrt{r}\left(\mathrm{e}^{-N r}-1\right) \sin \frac{\theta}{2}\right\|_{W_{2}^{2}\left(\Omega_{\delta}\right)} \leqslant C\|g\|_{L_{2}\left(\Omega_{\delta}\right)},
\end{aligned}
$$

where the constant $C$ is independent of $g, N$, and $\delta$. These estimates and (2.12) applied to the coefficients of the series (2.10) lead us to (2.8), (2.9), if we denote the fractions $\frac{\alpha}{\sqrt{N}}$ corresponding to $u_{j}$ and $\widetilde{u}_{j}$ by $a_{j}$ and $\widetilde{a}_{j}$. The inequality (1.3) can be checked by estimating the appropriate norms of $\sqrt{r} \mathrm{e}^{-N r}$ in the same manner as in (2.13).

Lemma 2.3. There exists $\delta_{0}>0$ small enough such that for any $f \in L_{2}\left(T_{\delta_{0}}\right)$ the generalized solution to

$$
\Delta_{x} u=f \quad \text { in } \quad T_{\delta_{0}}, \quad u=0 \quad \text { on } \quad \partial T_{\delta_{0}},
$$

satisfies (2.8), (2.9), (1.3).

Proof. Lemma 2.2 implies that the domain of the operator $\Delta_{\tau, x_{3}, s}$ in $T_{\delta}$ with Dirichlet boundary conditions is $\left\{u: u \in \mathfrak{V}_{\delta} \oplus W_{2}^{2}\left(T_{\delta}\right),\left.u\right|_{\partial T_{\delta}}=0\right\}$; the action of this operator reads as $\Delta_{\tau, x_{3}, s}^{(D)} u=\Delta_{\tau, x_{3}, s} u$. 
The Dirichlet Laplacian in $T_{\delta}$ can be written as

$$
\Delta_{x}^{(D)}=\Delta_{\tau, x_{3}, s}^{(D)}+\tau \frac{2 \mathrm{k}-\tau \mathbf{k}^{2}}{(1-\tau \mathrm{k})^{2}} \frac{\partial^{2}}{\partial s^{2}}+\mathcal{L}_{1} u, \quad \mathcal{L}_{1}=-\frac{\mathbf{k}}{1-\tau \mathbf{k}} \frac{\partial}{\partial \tau}-\frac{\tau \mathbf{k}^{\prime}}{(1-\tau \mathrm{k})^{3}} \frac{\partial}{\partial s},
$$

where $\mathbf{k}=\mathrm{k}(s) \in C^{\infty}(\partial \Omega)$. The operator $\tau \frac{2 \mathrm{k}-\tau \mathbf{k}}{(1-\tau \mathbf{k})^{2}} \frac{\partial^{2}}{\partial s^{2}}$ is $\Delta_{\tau, x_{3}, s^{-}}^{(D)}$ bounded due to (2.9), (1.3), and the bound is estimated by $C \delta, C$ is independent of $\delta$. The operator $\mathcal{L}_{1}$ is $\Delta_{\tau, x_{3}, s^{-}}^{(D)}$ compact. Employing now [11, Ch. IV, Sec. 1.1, Th. 1.1], we conclude that the domain of $\Delta_{x}^{(D)}$ is the same as that of $\Delta_{\tau, x_{3}, s}^{(D)}$, if $\delta$ is small enough. Therefore, the representation (2.8) is valid. The estimates (2.9), (1.3) for the solution to (2.14) follow from those for the solution to (2.7) and [11, Ch. IV, Sec. 1.4, Th. 1.16].

Let $u$ be a function in the domain of $\mathcal{H}_{\omega}$. By the definition, $u \in \stackrel{0}{W_{2}^{1}}\left(\Pi_{\omega}\right)$, and it is a generalized solution to

$$
-\Delta_{x} u=f \quad \text { in } \quad \Pi_{\omega}, \quad u=0 \text { on } \partial \Pi_{\omega},
$$

where $f=\mathcal{H}_{\omega} u$. Using the smoothness improving theorems one can make sure that $u \in W_{2}^{2}(S)$ for any $S \in \Pi_{\omega} \backslash T_{\delta}, \delta>0$, and hence

$$
\mathcal{H}_{\omega} u=-\Delta_{x} u \text {. }
$$

It is also clear that

$$
\|u\|_{W_{2}^{1}\left(T_{\delta}\right)} \leqslant C\|f\|_{L_{2}\left(T_{\delta}\right)} .
$$

We denote

$$
\widetilde{u}(x):=\left(1-\chi\left(\frac{r}{2 \delta}\right)\right) u(x) \in \stackrel{0}{W_{2}^{2}}\left(\Pi_{\omega}\right) .
$$

Employing (2.17) and proceeding as in the proof of Lemma 7.1 in [10, Ch. 3, Sec. $7]$, one can check that and

$$
\|\widetilde{u}\|_{W_{2}^{2}\left(\Pi_{\omega}\right)} \leqslant C\|f\|_{L_{2}\left(\Pi_{\omega}\right)} .
$$

The function $\widehat{u}(x):=u(x) \chi\left(\frac{r}{2 \delta}\right)$ is the solution to (2.14) with the right-hand side

$$
\tilde{f}:=-f-2 \nabla_{x} u \cdot \nabla_{x} \chi-u \Delta_{x} \chi, \quad \chi=\chi\left(\frac{r}{2 \delta}\right) .
$$

In view of (2.17) we have $\|\widetilde{f}\|_{L_{2}\left(T_{\delta}\right)} \leqslant C(\delta)\|f\|_{L_{2}\left(T_{\delta}\right)}$. Employing now Lemma 2.3, we conclude that the representation (2.8) and the estimates (2.9), (1.3) hold true. It remains to note that by (2.8)

$$
\widehat{u}=\chi\left(\frac{r}{\delta}\right) \widehat{u}=\chi\left(\frac{r}{\delta}\right) \widehat{u}^{(0)}+\chi\left(\frac{r}{\delta}\right) \widehat{u}^{(1)} .
$$

Denoting now $u^{(1)}:=\widetilde{u}+\chi\left(\frac{r}{\delta}\right) \widehat{u}^{(1)}$, we conclude that (1.1) holds true.

If $u$ is given by (1.1), it is easy to check that $u \in \stackrel{0}{W}_{2}^{1}\left(\Pi_{\omega}\right)$ is the generalized solution to the problem (2.15), where the right-hand side is that of (1.2). Thus, $u$ belongs to the domain of $\mathcal{H}_{\omega}$. To prove (1.2), it is sufficient to substitute (1.1) into (2.16). The proof of Theorem 1.1 is complete. 


\section{$3 \quad$ Estimates and continuity of the eigenvalues}

In the section we prove Theorems [1.2, 1.3,

Proof of Theorem 1.2. The main idea of the proof is borrowed from [5, Sec. II]. We introduce additional boundary $\partial \omega \times(-d, \pi)$ and impose in turn Dirichlet and Neumann boundary condition on it. As the result, we have two direct sum $\mathcal{H}_{\text {int }}^{(D)} \oplus$ $\mathcal{H}_{\text {ext }}^{(D)}$ and $\mathcal{H}_{\text {int }}^{(N)} \oplus \mathcal{H}_{\text {ext }}^{(N)}$, where $\mathcal{H}_{\text {int }}^{(D)}$ is the Dirichlet Laplacian in $\omega \times(-d, \pi)$, and $\mathcal{H}_{\text {ext }}^{(D)}$ is the Dirichlet Laplacian in $\Pi_{\omega} \backslash(\omega \times(-d, \pi))$. The operators $\mathcal{H}_{\text {int }}^{(N)}$, $\mathcal{H}_{\text {ext }}^{(N)}$ are introduced in the same way; the difference is the boundary condition on $\partial \omega \times(-d, \pi)$ which is the Neumann one.

The identities

$$
\sigma_{\text {ess }}\left(\mathcal{H}_{\omega}\right)=\sigma\left(\mathcal{H}_{\text {ext }}^{(D)}\right)=\sigma_{\text {ess }}\left(\mathcal{H}_{\text {ext }}^{(D)}\right)=\sigma\left(\mathcal{H}_{\text {ext }}^{(N)}\right)=\sigma_{\text {ess }}\left(\mathcal{H}_{\text {ext }}^{(N)}\right)=[1,+\infty)
$$

can be proven in the same way as the similar identity in the proof of Theorem 2.1 in [2]. The eigenvalues of $\mathcal{H}_{i n t}^{(N)}, \mathcal{H}_{\text {int }}^{(D)}$ are calculated by separation of variables,

$$
\lambda_{i, j}\left(\mathcal{H}_{\text {int }}^{(D)}\right)=\mu_{i}^{(D)}+\frac{\pi^{2} j^{2}}{(\pi+d)^{2}}, \quad \lambda_{i, j}\left(\mathcal{H}_{\text {int }}^{(N)}\right)=\mu_{i}^{(N)}+\frac{\pi^{2} j^{2}}{(\pi+d)^{2}}, \quad i, j \geqslant 1 .
$$

It is clear that $\pi^{2} j^{2} /(\pi+d)^{2}>1, j \geqslant 2$. Taking into account this inequality and standard bracketing [14, Ch. XIII, Sec. 15], we arrive at the estimates (1.4). The estimates (1.5) follow from (1.4).

Proof of Theorem 1.3. Let us prove first that the eigenvalues of $\mathcal{H}_{\omega(t)}$ are continuous w.r.t. $t$. Given $t_{0} \in(0,+\infty)$ and $t$ close to $t_{0}$, we introduce new variables by the rule

$$
\begin{aligned}
& \widetilde{x}=\left(\widetilde{x}^{\prime}, \widetilde{x}_{3}\right), \quad \widetilde{x}^{\prime}=\chi\left(\frac{r}{\delta}\right) \mathcal{M}\left(t_{0}, t\right) x^{\prime}+\left(1-\chi\left(\frac{r}{\delta}\right)\right) x^{\prime}, \\
& \widetilde{x}_{3}=\left(b\left(x^{\prime}, t\right) \chi\left(\frac{r}{\delta}\right)+1-\chi\left(\frac{r}{\delta}\right)\right) x_{3}, \quad b\left(x^{\prime}, t\right)=\sqrt{\left(\frac{\partial \widetilde{\tau}}{\partial \tau}\right)^{2}+\frac{1}{(1-\tau \mathrm{k})^{2}}\left(\frac{\partial \widetilde{s}}{\partial s}\right)^{2}}
\end{aligned}
$$

where $(\widetilde{\tau}, \widetilde{s})$ are associated w.r.t. with $\widetilde{x}^{\prime}$ in the same way as $(\tau, s)$ and $x^{\prime}$. Bearing in mind (A1), one can easily make sure that the variables $\widetilde{x}$ are well-defined for $t$ sufficiently close to $t_{0}$, and the domain $\Pi_{\omega\left(t_{0}\right)}$ is mapped onto $\Pi_{\omega(t)}$ under the change of variables. In the space $L_{2}\left(\Pi_{\omega\left(t_{0}\right)}\right)=L_{2}\left(\Pi_{\omega(t)}\right)$ we define a unitary operator

$$
(\mathcal{Q}(t) u)=\sqrt{q} u(Q(\cdot)), \quad q:=\operatorname{det}\left(\frac{\partial x_{i}}{\partial \widetilde{x}_{j}}\right)_{i, j=1, \ldots, 3},
$$

where $Q$ is defined as $x=Q(\widetilde{x})$. By direct calculations we check that

$$
\mathcal{Q}(t) \mathcal{H}_{\omega(t)} \mathcal{Q}^{-1}(t)=b\left(Q^{\prime}(\cdot), t\right) \mathcal{H}_{\omega\left(t_{0}\right)}+\mathcal{L}_{2},
$$




$$
\begin{aligned}
\mathcal{L}_{2}:= & b_{12} \frac{\partial^{2}}{\partial \tau \partial s}+x_{3} b_{13} \frac{\partial^{2}}{\partial \tau \partial x_{3}}+b_{22} \frac{\partial^{2}}{\partial s^{2}}+b_{23} \frac{\partial^{2}}{\partial s \partial x_{3}}+x_{3} b_{33} \frac{\partial^{2}}{\partial x_{3}^{2}} \\
& +b_{1} \frac{\partial}{\partial \tau}+b_{2} \frac{\partial}{\partial s}+b_{3} \frac{\partial}{\partial x_{3}}+b_{0},
\end{aligned}
$$

where $b_{i, j}=b_{i, j}(x, t) \in C\left(T_{\delta} \times\left(t_{0}-c, t_{0}+c\right)\right), b_{i}=b_{i}(x, t) \in C\left(T_{\delta} \times\left(t_{0}-c, t_{0}+c\right)\right)$, and $b_{i, j}(x, 0)=b_{i}(x, 0)=0$. The supports of $b_{i, j}, b_{i}$ lie inside $T_{\delta}$. It is also follows from (A1) that

$$
b\left(x^{\prime}, t\right)=1+\widetilde{b}\left(x^{\prime}, t\right), \quad \widetilde{b}\left(x^{\prime}, t\right) \in C_{0}\left(T_{\delta} \times\left(t_{0}-c, t_{0}+c\right)\right), \quad \widetilde{b}\left(x^{\prime}, 0\right)=0 .
$$

Theorem 1.1, and, in particular, the estimates (1.3) imply that $\mathcal{L}_{2}$ is $\mathcal{H}_{\omega\left(t_{0}\right)^{-}}$ bounded. By (3.1) and the last formula for $b$ we conclude now that the difference $\left(\mathcal{Q}(t) \mathcal{H}_{\omega(t)} \mathcal{Q}^{-1}(t)-\mathcal{H}_{\omega\left(t_{0}\right)}\right)$ is a small perturbation bounded relatively w.r.t. $\mathcal{H}_{\omega\left(t_{0}\right)}$, and $\left(\mathcal{Q}\left(t_{0}\right) \mathcal{H}_{\omega\left(t_{0}\right)} \mathcal{Q}^{-1}\left(t_{0}\right)=\mathcal{H}_{\omega\left(t_{0}\right)}\right)$. Thus, the eigenvalues of $\mathcal{Q}(t) \mathcal{H}_{\omega(t)} \mathcal{Q}^{-1}(t)$, and hence of $\mathcal{H}_{\omega(t)}$ converges to ones of $\mathcal{H}_{\omega\left(t_{0}\right)}$.

Assume now that $\omega\left(t_{1}\right) \subset \omega\left(t_{2}\right)$ for all $t_{1}<t_{2}$. These are the standard minimax arguments those show that the eigenvalues $\lambda_{i}(\omega(t))$ are monotonically decreasing functions of $t$. Hence, to prove the last statement of the theorem it is sufficient to show that for each eigenvalue $\lambda_{i}(\omega(t))$ there exists $t_{i}$ such that $\lambda_{i}(\omega(t)) \rightarrow 1-0$, as $t \rightarrow t_{i}+0$. Suppose that this is wrong for an eigenvalue $\lambda_{j}(\omega(t))$ on a sequence $t^{(m)} \rightarrow+0$. In this case $\lambda_{1}\left(\omega\left(t^{(m)}\right)\right) \leqslant \lambda_{j}\left(\omega\left(t^{(m)}\right)\right) \leqslant c<1$. At the same time, by [6. Th. 3.1] and the second identity in (1.6) we have $\lambda_{1}(\omega(t)) \rightarrow 1-0, t \rightarrow+0$, the contradiction. The sequence of critical values $t_{i}$ is infinite due to (1.4) and the first identity in (1.6).

\section{Reduction of the resolvent to a compact oper- ator}

In this section we study the boundary value problem

$$
-\Delta u=\left(1-k^{2}\right) u+f \quad \text { in } \quad \Pi_{\omega}, \quad u=0 \quad \text { on } \partial \Pi_{\omega},
$$

where $k \in \mathbb{C}$ ranges in a small neighbourhood of zero, $f \in L_{2}\left(\Pi_{\omega}\right)$, supp $f \subseteq$ $\Pi_{\omega, \beta}:=\left\{x:\left|x^{\prime}\right|<\beta\right\}, \beta>0$. We choose $\beta$ so that $\omega \subset\left\{x^{\prime}:\left|x^{\prime}\right|<\beta / 4\right\}$. If $d=\pi$, we assume in addition that $g$ is even w.r.t. $x_{3}$ and the same is for $u$. We should also specify the behaviour at infinity for the solutions. If $\operatorname{Re} k>0$, we take the function $u=\left(\mathcal{H}_{\omega}-1+k^{2}\right)^{-1} f$ as the solution to (4.1). For other values of $k$ we will define the analytic continuation of the operator $\left(\mathcal{H}_{\omega}-1+k^{2}\right)^{-1}$. We will do it by the technique employed in [1, Sec. 3], [3, Sec. 3.A]. We will also reduce (4.1) to a Fredholm equation in $L_{2}\left(\Pi_{\omega, \beta}\right)$ that will be one of the key ingredient in the proof of Theorems 1.5, 1.6.

Let $g \in L_{2}\left(\Pi_{\omega, \beta}\right)$, supp $g \subseteq \Pi_{\omega, \beta}$, and $g$ is even w.r.t. $x_{3}$, if $d=\pi$. By $v=v(x, k)$ we denote the solution to the problem

$$
-\Delta v=\left(1-k^{2}\right) v+g \quad \text { in } \quad \Pi_{\emptyset}, \quad v=0 \quad \text { on } \partial \Pi_{\emptyset},
$$


given by the formulas

$$
\begin{aligned}
& v(x, k)= \begin{cases}v^{+}(x, k), & x_{3} \in(0, \pi), \\
v^{-}(x, k), & x_{3} \in(-d, 0),\end{cases} \\
& v^{+}(x, k)=\sum_{j=1}^{\infty} v_{j}^{+}\left(x^{\prime}, k\right) \sin j x_{3}, \quad v^{-}(x, k)=\sum_{j=1}^{\infty} v_{j}^{-}\left(x^{\prime}, k\right) \sin \frac{\pi j}{d} x_{3},
\end{aligned}
$$

where $\mathrm{H}_{0}$ is the Hankel function, and $\sqrt{k^{2}}=k$, while the other roots are specified by the requirement $\sqrt{1}=1$. If $k=0$, we introduce the function $v_{1}^{+}$as

$$
v_{1}^{+}\left(x^{\prime}, 0\right)=\frac{1}{\pi^{2}} \int_{\mathbb{R}^{2} \times(0, \pi)} g(y) \ln \left|x^{\prime}-y^{\prime}\right| \sin y_{3} \mathrm{~d} y,
$$

and

$$
v_{1}^{+}\left(x^{\prime}, 0\right)=\frac{1}{\pi^{2}} \int_{\mathbb{R}^{2} \times(-\pi, 0)} g(y) \ln \left|x^{\prime}-y^{\prime}\right| \sin y_{3} \mathrm{~d} y, \quad \text { if } \quad d=\pi .
$$

The function $v$ is well-defined and belongs to $W_{2}^{2}\left(\Pi_{\emptyset, \widetilde{\beta}}\right)$ for all $\widetilde{\beta}>0$ and considered values of $k$. This fact can be shown by analogy with the proof of Lemma 3.1 in [1].

Consider the problem

$$
\Delta w=\Delta v \quad \text { in } \quad \Pi_{\omega}, \quad w=v \quad \text { on } \quad \partial \Pi_{\omega} .
$$

This problem is uniquely solvable in $W_{2}^{1}\left(\Pi_{\omega, \beta}\right)$. We construct the solution to (4.1) as

$$
u(x, k)=\left(\mathcal{A}_{1}(k) g\right)(x, k):=w(x, k) \chi\left(\frac{\left|x^{\prime}\right|}{\beta}\right)+\left(1-\chi\left(\frac{\left|x^{\prime}\right|}{\beta}\right)\right) v(x, k) .
$$

This function satisfies the boundary condition in (4.1). Substituting it into the equation in (4.1), we obtain

$$
\begin{gathered}
g+\mathcal{A}_{2}(k) g=f \\
\mathcal{A}_{2}(k) g:=(v-w)\left(\Delta+1-k^{2}\right) \chi\left(\frac{\left|x^{\prime}\right|}{\beta}\right)+2 \nabla \chi\left(\frac{\left|x^{\prime}\right|}{\beta}\right) \cdot \nabla(v-w) .
\end{gathered}
$$

By $\mathfrak{A}$ we denote the set of the operators $\mathcal{A}=\mathcal{A}(k)$ bounded as ones from $L_{2}\left(\Pi_{\omega, \beta}\right)$ into $W_{2}^{1}\left(\Pi_{\omega, \widetilde{\beta}}\right), \mathfrak{W}(\delta, \widetilde{\beta})$ and $W_{2}^{2}\left(\Pi_{\omega, \widetilde{\beta}} \backslash T_{\delta}\right)$ for each $\widetilde{\beta}>0, \delta>0$, and small 
$k$, and such that the function $\mathcal{A}(k) f$ is real-valued for real-valued $f$ and small nonnegative $k$. If an operator $\mathcal{A}(k)$ belongs to $\mathfrak{A}$ and is continuous (uniformly bounded, holomorphic) w.r.t. $k$ as an operator from $L_{2}\left(\Pi_{\beta}\right)$ into each of aforementioned spaces, we will say shortly that the operator $\mathcal{A}(k)$ belongs to $\mathfrak{A}$ and is continuous (uniformly bounded, holomorphic) w.r.t. $k$.

We denote

$$
\mathrm{a}(g):=\frac{1}{\pi^{2}} \int_{\Pi_{\omega, \beta} \cap\left\{x: x_{3}>0\right\}} g(x) \sin x_{3} \mathrm{~d} x_{3} .
$$

Repeating the arguments of the proofs of Lemmas 3.1, 3.3, 3.4, 3.5 in [1] and of Propositions 3.1, 3.2 in [3], and employing Lemma 2.3] one can prove

Lemma 4.1. Let $k \in \mathbb{C}$ be small enough. The operator $\mathcal{A}_{1}(k) \in \mathfrak{A}$ is bounded uniformly w.r.t. small $k$. The operator $\mathcal{A}_{2}(k)$ is a linear compact operator in $L_{2}\left(\Pi_{\omega, \beta}\right)$. For $k \neq 0$ it can be represented as

$$
\mathcal{A}_{1}(k)=\mathcal{A}_{2}\left(k^{2}\right)+\mathcal{A}_{3}\left(k^{2}\right) \ln k, \quad \mathcal{A}_{2}(k)=\mathcal{A}_{5}\left(k^{2}\right)+\mathcal{A}_{6}\left(k^{2}\right) \ln k,
$$

where $\mathcal{A}_{3}(\cdot), \mathcal{A}_{4}(\cdot) \in \mathfrak{A}$ are holomorphic, and $\mathcal{A}_{5}(\cdot), \mathcal{A}_{6}(\cdot)$ are linear compact operators in $L_{2}\left(\Pi_{\omega, \beta}\right)$ being holomorphic w.r.t. $k$. The functions $\mathcal{A}_{i}\left(k^{2}\right) f, i=5,6$, are real-valued if $f$ is real-valued and $k^{2}$ is small and non-negative. For each $f \in L_{2}\left(\Pi_{\omega, \beta}\right)$ there exists a solution to (4.1) given by $u=\mathcal{A}_{1}(k) g$. This solution behaves at infinity as

$$
\begin{aligned}
& u(x, k)=\mathrm{c}\left(k, \frac{x^{\prime}}{\left|x^{\prime}\right|}\right) \mathrm{e}^{-k\left|x^{\prime}\right|}\left|x^{\prime}\right|^{-\frac{1}{2}} \sin x_{3}+\mathcal{O}\left(\mathrm{e}^{-k\left|x^{\prime}\right|}\left|x^{\prime}\right|^{-\frac{3}{2}}\right), \quad x_{3} \in(0, \pi), \\
& \mathrm{c}(k, \xi)=-\frac{\sqrt{2 \pi}}{4 \sqrt{k}} \mathrm{a}\left(\left(1+k \xi \cdot x^{\prime}\right) g(x)\right)+\mathcal{O}\left(k^{\frac{3}{2}}\right), \quad k \rightarrow 0, \\
& u(x, k)=\mathcal{O}\left(\mathrm{e}^{-\sqrt{\frac{\pi^{2}}{d^{2}}-1+k^{2}}\left|x^{\prime}\right|}\left|x^{\prime}\right|^{-\frac{3}{2}}\right), \quad x_{3} \in(-d, 0), \quad \text { if } \quad d<\pi,
\end{aligned}
$$

if $k \neq 0$, and

$$
\begin{aligned}
& u(x, 0)=\left(\mathrm{a}(g) \ln \left|x^{\prime}\right|+\frac{c_{1} x_{1}+c_{2} x_{2}}{\left|x^{\prime}\right|^{2}}\right) \sin x_{3}+\mathcal{O}\left(\left|x^{\prime}\right|^{-2}\right), \quad x_{3} \in(0, \pi), \\
& u(x, 0)=\mathcal{O}\left(\mathrm{e}^{-\sqrt{\frac{\pi^{2}}{d^{2}}-1}\left|x^{\prime}\right|}\left|x^{\prime}\right|^{-\frac{3}{2}}\right), \quad x_{3} \in(-d, 0), \quad \text { if } \quad d<\pi .
\end{aligned}
$$

For each solution to (4.1) behaving at infinity in accordance with (4.7), (4.8) there exists the unique solution to (4.4) such that $u=\mathcal{A}_{1}(k) g$.

We denote

$$
V_{0}(x):= \begin{cases}\sin x_{3}, & x_{3} \in(0, \pi), \\ (1-\gamma) \sin x_{3}, & x_{3} \in(-d, 0) .\end{cases}
$$

By $W_{0}$ we indicate the solution to (4.2) as $v=V_{0}$. We introduce the functions $U_{0}$, by (4.3) via $V_{0}$ and $W_{0}$. The next lemma is checked by direct calculations. 
Lemma 4.2. The identities

$$
\begin{array}{ll}
\mathcal{A}_{3}(0) g=(\mathrm{C}-\ln 2) \mathcal{A}_{4}(0) g+\mathcal{A}_{1}(0) g, & \mathcal{A}_{4}(0) g=\mathrm{a}(g) U_{0}, \\
\mathcal{A}_{5}(0) g=(\mathrm{C}-\ln 2) \mathcal{A}_{6}(0) g+\mathcal{A}_{2}(0) g, & \mathcal{A}_{6}(0) g=-\mathrm{a}(g)(\Delta+1) U_{0},
\end{array}
$$

hold true.

Lemma 4.3. Let $k=0$. There is a finite number of linear independent non-trivial solutions to (4.1), (4.8) assumed to be even w.r.t. to $x_{3}$ if $d=\pi$. They can be chosen so that there is at most one solution behaving at infinity

$$
\Psi(x)=\ln \left|x^{\prime}\right| \sin x_{3}+\mathcal{O}\left(\left|x^{\prime}\right|^{-1}\right), \quad\left|x^{\prime}\right| \rightarrow+\infty, \quad x_{3} \in(0, \pi) ;
$$

at most two solutions satisfying (1.9), and a finite number of solutions belonging to $L_{2}\left(\Pi_{\omega}\right)$. Each of these solutions is infinitely differentiable up to the boundary except $\partial \omega \times\{0\}$, and in the vicinity of $\partial \omega \times\{0\}$ it satisfies (1.10).

Proof. The statement on the existence and the number of the solutions follows immediately from (4.8). The claimed smoothness is due to the standard smoothness improving theorems. The formula (1.10) can be checked by analogy with the proof of Lemma 4.2 in [1].

Lemma 4.4. Let $k=0$. The equation $(\sqrt{4.4})$ is solvable if and only if $\left(f, \Psi_{i}\right)_{L_{2}\left(\Pi_{\omega, \beta}\right)}=$ 0 , where $\Psi_{i}$ are non-trivial solutions to (4.1), (4.8). If the solvability conditions holds true, there exists the unique solution of (4.4) orthogonal to $\phi$.

Proof. By Lemma 4.1 the operator $\mathcal{A}_{2}(0)$ is compact. Thus, the equation (4.4) is solvable if and only if $\left(f, \phi_{i}^{*}\right)_{L_{2}\left(\Pi_{\omega, \beta}\right)}=0$, where $\phi_{i}^{*}$ are non-trivial solutions to the adjoint equation $\phi_{i}^{*}+\mathcal{A}_{2}^{*}(0) \phi_{i}^{*}=0$. It is sufficient to show that $\phi_{i}^{*}=\Psi_{i}$. Since the number of $\phi_{i}^{*}$ and $\Psi_{i}$ are the same, in view of Lemma 4.1 it is sufficient to check that

$$
0=\left(\Psi_{i}+\mathcal{A}_{2}^{*}(0) \Psi_{i}, h\right)_{L_{2}\left(\Pi_{\omega, \beta}\right)}=\left(\Psi_{i}, h+\mathcal{A}_{2}(0) h\right)_{L_{2}\left(\Pi_{\omega, \beta}\right)}
$$

for all $h \in L_{2}(\omega, \beta)$. We denote $u:=\mathcal{A}_{1}(0) h$; by the definition of $\mathcal{A}_{1}(0)$ this function satisfies (4.8). The same formula is valid for $\Psi_{i}$. Moreover, $h+\mathcal{A}_{2}(0) h=$ $-(\Delta+1) u$. Taking these facts into account and integrating by parts, we obtain

$$
\left(\Psi_{i}, h+\mathcal{A}_{2}(0) h\right)_{L_{2}\left(\Pi_{\omega, \beta}\right)}=-\int_{\Pi_{\omega, \beta}} \Psi_{i}(\Delta+1) u \mathrm{~d} x=-\int_{\Pi_{\omega}} u(\Delta+1) \Psi_{i} \mathrm{~d} x=0 .
$$

Proof of Lemma 1.4. The most part of the lemma follows from Lemma 4.3, it remains to check the statement on the solution satisfying (1.8). If there exists the non-trivial solution $u$ behaving at infinity in accordance with (4.9), the problem (1.7) can not has a solution $\Psi$ satisfying (1.8). This fact can be proven by integrating by parts in the integral $0=\int_{\Omega_{\beta}} u(\Delta+1) \Psi \mathrm{d} x$. 
Assume that there is no non-trivial solution obeying (4.9); let us prove that in this case there is the unique non-trivial solution behaving at infinity as

$$
\Psi(x)=\left(c \ln \left|x^{\prime}\right|+1\right) \sin x_{3}+\mathcal{O}\left(\left|x^{\prime}\right|^{-1}\right), \quad\left|x^{\prime}\right| \rightarrow+\infty, \quad x_{3} \in(0, \pi), \quad c \neq 0 .
$$

We construct it as

$$
\begin{array}{llll}
\Psi(x)=\widetilde{\Psi}(x)+\left(1-\chi\left(\frac{2\left|x^{\prime}\right|}{3 \beta}\right)\right) \ln \left|x^{\prime}\right| \sin x_{3}, & x_{3} \in(0, \pi), & \\
\Psi(x)=\widetilde{\Psi}(x), & x_{3} \in(-d, 0), \quad \text { if } \quad d<\pi, \\
\Psi(x)=\widetilde{\Psi}(x)+\left(1-\chi\left(\frac{2\left|x^{\prime}\right|}{3 \beta}\right)\right) \ln \left|x^{\prime}\right| \sin x_{3}, & x_{3} \in(-\pi, 0), \quad \text { if } \quad d=\pi .
\end{array}
$$

It leads us to the problem (4.1) for $\widetilde{\Psi}$ with $k=0$ where

$$
\begin{array}{ll}
f(x)=(\Delta+1)\left(1-\chi\left(\frac{2\left|x^{\prime}\right|}{3 \beta}\right)\right) \sin x_{3}, & x_{3} \in(0, \pi), \\
f(x)=(\gamma-1)(\Delta+1)\left(1-\chi\left(\frac{2\left|x^{\prime}\right|}{3 \beta}\right)\right) \sin x_{3}, & x_{3} \in(-\pi, 0) .
\end{array}
$$

Taking into account Lemma 4.1 and integrating by parts, it is easy to check that $\left(f, \Psi_{i}\right)_{L_{2}\left(\Pi_{\omega, \beta}\right)}=0$ for each non-trivial solution $\Psi_{i}$ of (4.1), (4.8). By Lemma 4.4 the equation (4.4) is thus solvable that by Lemma 4.1 proves the solvability of the problem for $\widetilde{\Psi}$. The uniqueness of $\Psi$ follows from (4.10).

\section{Singularity of the resolvent}

In this section we study the behaviour of the operator $\left(\mathrm{I}+\mathcal{A}_{2}(k)\right)^{-1}$ in the vicinity of $k=0$. We remind, that if $d=\pi$, we restrict all the operators on the even w.r.t. $x_{3}$ functions. We consider two cases corresponding to different possibilities of presence of non-trivial solution described in Lemmas 1.4, 4.3. The results of the section will be employed in the proof of Theorems 1.5, 1.6.

\subsection{Absence of decaying and logarithmically growing non- trivial solution}

Here we deal with the case when the problem (4.1), (4.8) has no non-trivial solutions described in Lemma 4.3. As it has been shown in the proof of Lemma 1.4, in this case the problem (1.7) has the unique non-trivial solution satisfying (4.10).

We substitute (4.6) into (4.4) and take into account Lemma 4.2. It leads us to

$$
g+\mathcal{A}_{2}(0) g-(\ln k-\ln 2+\mathrm{C}) \mathrm{a}(g)(\Delta+1) U_{0}+k^{2} \ln k \mathcal{A}_{7}(k) g=f,
$$

where

$$
\mathcal{A}_{7}(k):=\frac{\mathcal{A}_{5}\left(k^{2}\right)-\mathcal{A}_{5}(0)+\left(\mathcal{A}_{6}\left(k^{2}\right)-\mathcal{A}_{6}(0)\right) \ln k}{k^{2} \ln k}
$$


is a compact operator in $L_{2}\left(\Pi_{\omega, \beta}\right)$ continuous w.r.t. small real $k$. The same is true for $\left(k \mathcal{A}_{7}(k)\right)^{\prime}$. Hereinafter the expressions like $\ln k \mathcal{A}_{7}(k)$ are understood as $(\ln k) \mathcal{A}_{7}(k)$.

By the assumption the operator $\left(\mathrm{I}+\mathcal{A}_{2}(0)\right)$ is invertible, and the same is thus true for $\left(\mathrm{I}+\mathcal{A}_{2}(0)+k^{2} \ln k \mathcal{A}_{7}(k)\right)$. We denote the inverse to the latter as $\mathcal{A}_{8}(k)$ and apply it to (5.1):

$$
g-(\ln k-\ln 2+\mathrm{C}) \mathrm{a}(g) \mathcal{A}_{8}(k)(\Delta+1) U_{0}=\mathcal{A}_{8}(k) f,
$$

Now we apply the functional a to the equation obtained that solves (5.1):

$$
\begin{gathered}
\mathrm{a}(g)=\frac{\mathrm{a}\left(\mathcal{A}_{8}(k) f\right)}{1-(\ln k-\ln 2+\mathrm{C}) \mathrm{a}\left(\mathcal{A}_{8}(k)(\Delta+1) U_{0}\right)}, \\
\left(\mathrm{I}+\mathcal{A}_{2}(k)\right)^{-1} f=g=\frac{(\ln k-\ln 2+\mathrm{C}) \mathrm{a}\left(\mathcal{A}_{8}(k) f\right) \mathcal{A}_{8}(k)(\Delta+1) U_{0}}{1-(\ln k-\ln 2+\mathrm{C}) \mathrm{a}\left(\mathcal{A}_{8}(k)(\Delta+1) U_{0}\right)}+\mathcal{A}_{8}(k) f .
\end{gathered}
$$

Let us prove that the denominator is non-zero. In order to do it, we need

Lemma 5.1. The identities

$$
\mathcal{A}_{1}(0) \mathcal{A}_{8}(0)(\Delta+1) U_{0}+U_{0}=\Psi, \quad \mathrm{a}\left((\Delta+1) U_{0}\right)=c,
$$

hold true, where $\Psi$ is the unique solution to (1.7), (1.8), and $c$ is from (4.10).

Proof. It is sufficient to prove the former formula, since it implies the latter due to (4.8).

The definition of $\mathcal{A}_{8}$ yields that $\mathcal{A}_{8}(0)(\Delta+1) U_{0}=\left(\mathrm{I}+\mathcal{A}_{2}(0)\right)^{-1}(\Delta+1) U_{0}$, and hence $\mathcal{A}_{8}(0)(\Delta+1) U_{0}$ is the function $g$ corresponding to the solution $u$ of (4.1), (4.9) with $k=0, f=(\Delta+1) U_{0}$. Thus, the function $u+U_{0}$ solves (1.7), (1.8), that by the uniqueness completes the proof.

This lemma and the definition of $\mathcal{A}_{8}$ imply that

$$
\mathrm{a}\left(\mathcal{A}_{8}(k)(\Delta+1) U_{0}\right)=c+k^{2} h(k) \ln k,
$$

where the function $h(k)$ is continuous w.r.t. small real $k$, and the same is true for $(k h(k))^{\prime}$. We substitute this identity into (5.2), take into account (4.6) and Lemmas 4.2, 5.1, and arrive at

Lemma 5.2. If the problem (1.7) has no bounded non-trivial solution satisfying (4.8) or (1.8), then the operator $\mathcal{A}_{1}(k)\left(\mathrm{I}+\mathcal{A}_{2}(k)\right)^{-1} \in \mathfrak{A}$ is bounded uniformly in small real $k$. If the problem (1.7) has the unique bounded non-trivial solution and it satisfies (1.8), then

$$
\begin{aligned}
\mathcal{A}_{1}(k)\left(\mathrm{I}+\mathcal{A}_{2}(k)\right)^{-1}= & (\ln k-\ln 2+\mathrm{C}) \mathrm{a}\left(\left(\mathrm{I}+\mathcal{A}_{2}(0)\right)^{-1} \cdot\right) \Psi \\
& +\mathcal{A}_{1}(0)\left(\mathrm{I}+\mathcal{A}_{2}(0)\right)^{-1}+k^{2} \ln ^{3} k \mathcal{A}_{9}(k),
\end{aligned}
$$

where $\mathcal{A}_{9} \in \mathfrak{A}$ is continuous w.r.t. small real $k$ and the same is true for $\left(k \mathcal{A}_{9}(k)\right)^{\prime}$. 


\subsection{Presence of the unique logarithmically growing solu- tion}

In this subsection we study the case when the problem (1.7) has the unique solution satisfying (4.9). We denote it by $\Psi$; let $\phi \in L_{2}\left(\Pi_{\omega, \beta}\right)$ be the associated solution to (4.4) with $k=0, f=0$.

We construct the solution to (4.4) as

$$
g=\alpha \phi+\widetilde{g}
$$

where $\alpha=\alpha(\widetilde{g}, f)$ is a constant. We substitute this identity and (4.6) into (4.4) that yields

$$
\begin{aligned}
& \widetilde{g}+\mathcal{A}_{2}(0) \widetilde{g}-(\ln k-\ln 2+\mathrm{C}) \mathrm{a}(\widetilde{g})(\Delta+1) U_{0}+k^{2} \ln k \mathcal{A}_{7}(k) \widetilde{g} \\
& \quad-\alpha(\widetilde{g}, f)(\ln k-\ln 2+C)(\Delta+1) U_{0}+\alpha(\widetilde{g}, f) k^{2} \ln k \mathcal{A}_{7}(k) \phi=f .
\end{aligned}
$$

Here we have used the identity

$$
\mathrm{a}(\phi)=1
$$

which follows from the definition of $\phi$, and (4.8), (4.9). Integrating by parts, one can check that

$$
\int_{\Pi_{\omega, \beta}} \Psi(\Delta+1) U_{0} \mathrm{~d} x=-\gamma \pi^{2} .
$$

Taking into account this formula and Lemma 4.4, we calculate the inner product of (5.4) and $\Psi$,

$$
\begin{aligned}
\gamma \pi^{2}(\ln k-\ln 2+\mathrm{C})(\mathrm{a}(\widetilde{g}) & +\alpha(\widetilde{g}, f))+k^{2}\left(\mathcal{A}_{7}(k) \widetilde{g}, \Psi\right)_{L_{2}\left(\Pi_{\omega, \beta}\right)} \ln k \\
& +\alpha(\widetilde{g}, f) k^{2}\left(\mathcal{A}_{7}(k) \phi, \Psi\right)_{L_{2}\left(\Pi_{\omega, \beta}\right)} \ln k=(f, \Psi)_{L_{2}\left(\Pi_{\omega, \beta}\right)}
\end{aligned}
$$

Hence,

$$
\begin{aligned}
\alpha(\widetilde{g}, f)= & \frac{(f, \Psi)_{L_{2}\left(\Pi_{\omega, \beta}\right)}}{\gamma \pi^{2}(\ln k-\ln 2+\mathrm{C})+k^{2} \ln k\left(\mathcal{A}_{7}(k) \phi, \Psi\right)_{L_{2}\left(\Pi_{\omega, \beta}\right)}} \\
& -\frac{\gamma \pi^{2}(\ln k-\ln 2+\mathrm{C}) \mathrm{a}(\widetilde{g})+k^{2}\left(\mathcal{A}_{7}(k) \widetilde{g}, \Psi\right)_{L_{2}\left(\Pi_{\omega, \beta}\right)} \ln k}{\gamma \pi^{2}(\ln k-\ln 2+\mathrm{C})+k^{2}\left(\mathcal{A}_{7}(k) \phi, \Psi\right)_{L_{2}\left(\Pi_{\omega, \beta}\right)} \ln k} .
\end{aligned}
$$

We substitute this identity into (5.4) and obtain

$$
\begin{gathered}
\widetilde{g}+\mathcal{A}_{2}(0) \widetilde{g}+k^{2} \ln k \mathcal{A}_{10}(k) \widetilde{g}=\widetilde{f} \\
\mathcal{A}_{10}(k):=\mathcal{A}_{7}(k)-\frac{\gamma \pi^{2}(\ln k-\ln 2+\mathrm{C}) \mathrm{a}(\cdot)+k^{2}\left(\mathcal{A}_{7}(k) \cdot,\right)_{L_{2}\left(\Pi_{\omega, \beta}\right)} \ln k}{\gamma \pi^{2}(\ln k-\ln 2+\mathrm{C})+k^{2}\left(\mathcal{A}_{7}(k) \phi, \Psi\right)_{L_{2}\left(\Pi_{\omega, \beta}\right)} \ln k} \mathcal{A}_{7}(k) \phi \\
+\frac{\left(\mathcal{A}_{7}(k) \cdot, \Psi\right)_{L_{2}\left(\Pi_{\omega, \beta}\right)}-\mathrm{a}(\cdot)\left(\mathcal{A}_{7}(k) \phi, \Psi\right)_{L_{2}\left(\Pi_{\omega, \beta}\right)}}{\gamma \pi^{2}(\ln k-\ln 2+\mathrm{C})+k^{2} \ln k\left(\mathcal{A}_{7}(k) \phi, \Psi\right)_{L_{2}\left(\Pi_{\omega, \beta}\right)}}(\ln k-\ln 2+\mathrm{C})(\Delta+1) U_{0},
\end{gathered}
$$




$$
\tilde{f}=f+\frac{\left((\ln k-\ln 2+\mathrm{C})(\Delta+1) U_{0}-k^{2} \mathcal{A}_{7}(k) \phi \ln k\right)}{\gamma \pi^{2}(\ln k-\ln 2+\mathrm{C})+k^{2}\left(\mathcal{A}_{7}(k) \phi, \Psi\right)_{L_{2}\left(\Pi_{\omega, \beta}\right)} \ln k}(f, \Psi)_{L_{2}\left(\Pi_{\omega, \beta}\right)} .
$$

It is easy to check that the operator $\mathcal{A}_{10}$ is a compact one in $L_{2}\left(\Pi_{\omega, \beta}\right)$ bounded uniformly in small real $k$. One can also make sure that

$$
(\widetilde{f}, \Psi)_{L_{2}\left(\Pi_{\omega, \beta}\right)}=0, \quad\left(\mathcal{A}_{10}(k) \widetilde{g}, \Psi\right)_{L_{2}\left(\Pi_{\omega, \beta}\right)}=0 \quad \text { for each } \quad \widetilde{g} \in L_{2}\left(\Pi_{\omega, \beta}\right) .
$$

Hence, $f \in\{\Psi\}^{\perp}$, where $\{\Psi\}^{\perp}$ is the orthogonal complement to $\Psi$ in $L_{2}\left(\Pi_{\omega, \beta}\right)$. Since the operator $\left(\mathrm{I}+\mathcal{A}_{2}(0)\right)^{-1}:\{\Psi\}^{\perp} \rightarrow\{\phi\}^{\perp}$ is bounded by Lemma 4.4, we conclude that the operator $\left(\mathrm{I}+\mathcal{A}_{2}(0)+k^{2} \ln k \mathcal{A}_{10}(k)\right)^{-1}\{\Psi\}^{\perp} \rightarrow\{\phi\}^{\perp}$ is bounded uniformly in small real $k$. Thus,

$$
\widetilde{g}=\left(\mathrm{I}+\mathcal{A}_{2}(0)+k^{2} \ln k \mathcal{A}_{10}(k)\right)^{-1} .
$$

We also note that by (5.5), (5.6)

$$
\begin{aligned}
\mathrm{a}(g)= & \alpha(\widetilde{g})+\mathrm{a}(\widetilde{g}, f)=\frac{(f, \Psi)_{L_{2}\left(\Pi_{\omega, \beta}\right)}}{\gamma \pi^{2}(\ln k-\ln 2+\mathrm{C})+k^{2} \ln k\left(\mathcal{A}_{7}(k) \phi, \Psi\right)_{L_{2}\left(\Pi_{\omega, \beta}\right)}} \\
& +\frac{k^{2}\left(\mathcal{A}_{7}(k)(\mathrm{a}(\widetilde{g}) \phi \ln k-\widetilde{g}), \Psi\right)_{L_{2}\left(\Pi_{\omega, \beta}\right)}}{\gamma \pi^{2}(\ln k-\ln 2+\mathrm{C})+k^{2}\left(\mathcal{A}_{7}(k) \phi, \Psi\right)_{L_{2}\left(\Pi_{\omega, \beta}\right)} \ln k} .
\end{aligned}
$$

These identities, the formulas for $\alpha(\widetilde{g}, f)$ and $\widetilde{f}$, (5.3) , and Lemma 4.2 lead us to

Lemma 5.3. Suppose the problem (1.7) has the unique non-trivial solution, and it satisfies (4.9). Then the operator $\mathcal{A}_{1}(k)\left(\mathrm{I}+\mathcal{A}_{2}(k)\right)^{-1} \in \mathfrak{A}$ is bounded uniformly in small real $k$.

\section{Eigenvalues emerging from the essential spec- trum}

In the section we prove Theorems [1.5, 1.6,

Proof of Theorem 1.5. Given $\omega_{\varepsilon}$, we describe the domain $\Pi_{\omega_{\varepsilon}}$ in terms of the Cartesian coordinates $\widetilde{x}=\left(\widetilde{x}^{\prime}, \widetilde{x}_{3}\right)$, and introduce new variables as

$$
x=\chi\left(\frac{\widetilde{r}}{\delta}\right) \mathcal{M}(\varepsilon) \widetilde{x}+\left(1-\chi\left(\frac{\widetilde{r}}{\widetilde{\delta}}\right)\right) \widetilde{x}
$$

where $\widetilde{r}:=\sqrt{\widehat{\tau}^{2}+\widetilde{x}_{3}^{2}},(\widetilde{\tau}, \widetilde{s})$ are associated with $\widetilde{x}^{\prime}$ and $\partial \omega, \widetilde{\delta}>0$ is small enough. The mapping $\mathcal{M}(\varepsilon)$ is described by the formulas

$$
\tau=\widetilde{\tau}-\varepsilon \beta(s), \quad \widetilde{s}=s, \quad x_{3}=\widetilde{x}_{3} \sqrt{1+\frac{\varepsilon^{2}\left(\beta^{\prime}(s)\right)^{2}}{(1-\widetilde{\tau} \mathrm{k}(s))^{2}}} .
$$


It is clear that under this change of variables the domain $\Pi_{\omega_{\varepsilon}}$ is mapped onto $\Pi_{\omega}$.

We rewrite the eigenvalue equation $\mathcal{H}_{\omega_{\varepsilon}} \psi=\lambda \psi$ in the variables $x$ that leads us to

$$
\left(\mathcal{H}_{\omega}-\varepsilon \mathcal{L}_{3}(\varepsilon)\right) \psi=\lambda \psi
$$

where $\mathcal{L}_{3}(\varepsilon)$ is given by the expression in the right-hand side of (3.2) with the coefficients belonging to $C\left(T_{\delta} \times\left[0, \varepsilon_{0}\right]\right), \varepsilon_{0}>0$, and supports lying inside $T_{\delta}$. The operator $\mathcal{L}_{3}$ can be represented as

$$
\begin{aligned}
\mathcal{L}_{3}(\varepsilon) & =\mathcal{L}_{4}+\varepsilon \mathcal{L}_{5}+\varepsilon^{2} \mathcal{L}_{6}(\varepsilon), \quad \mathcal{L}_{4}=\beta \chi \frac{\partial}{\partial \tau}(\Delta+1)-(\Delta+1) \beta \chi \frac{\partial}{\partial \tau} \\
\mathcal{L}_{5}= & \frac{1}{2} \beta^{2} \frac{\partial}{\partial \tau} \chi^{2} \frac{\partial}{\partial \tau}(\Delta+1)+\frac{1}{2}(\Delta+1) \beta^{2} \chi^{2} \frac{\partial^{2}}{\partial \tau^{2}}-\beta \chi \frac{\partial}{\partial \tau}(\Delta+1) \beta \chi \frac{\partial}{\partial \tau} \\
& -\frac{\left(\beta^{\prime}\right)^{2} x_{3} \chi}{2(1-\tau \mathrm{k})^{2}} \frac{\partial}{\partial x_{3}}(\Delta+1)+(\Delta+1) \frac{\left(\beta^{\prime}\right)^{2} x_{3} \chi}{2(1-\tau \mathrm{k})^{2}} \frac{\partial}{\partial x_{3}}
\end{aligned}
$$

where $\beta=\beta(s), \chi=\chi(r / \widetilde{\delta})$, and $\mathcal{L}_{6}(\varepsilon)$ is given by the expression in the righthand side of (3.2) with the coefficients belonging to $C\left(T_{\delta} \times\left[0, \varepsilon_{0}\right]\right), \varepsilon_{0}>0$ and supports lying inside $T_{\delta}$. The operators $\mathcal{L}_{4}, \mathcal{L}_{5}$ are in fact second order differential operators satisfying (3.2) with some compactly supported continuous coefficients. We write them in terms of Laplace operators since it is more convenient for the following arguments. We also observe that the operators $\mathcal{L}_{i}, i=3,4,5$, are $\mathcal{H}_{\omega^{-}}$ bounded by Theorem 1.1 and the bounds can be estimated uniformly in $\varepsilon$.

We can rewrite (6.2) as

$$
\left(\mathcal{H}_{\omega}-\lambda\right) \psi=\varepsilon \mathcal{L}_{3} \psi
$$

If we denote now $\lambda=1-k^{2}$, we conclude that an eigenfunction $\psi$ is a non-trivial solution to (4.1), (4.7) with $f=f_{\varepsilon}:=\varepsilon \mathcal{L}_{3} \psi$.

Let us find all values of $k$ converging to zero as $\varepsilon \rightarrow+0$ for which the problem (4.1), (4.7) with $f=\varepsilon \mathcal{L}_{3} \psi$ has a non-trivial solution. If the solution belongs to $\mathcal{D}\left(\mathcal{H}_{\omega}\right)$, it will imply that $\lambda=1-k^{2}$ is an eigenvalue of $\mathcal{H}_{\omega_{\varepsilon}}$ close to the threshold of the essential spectrum. In order to find such values, we employ the approach similar to that used in [3], [1], [7], [8].

We note that in the case $d=\pi$ the eigenfunctions of $\mathcal{H}_{\omega_{\varepsilon}}$ are even w.r.t. $x_{3}$ that can be proved by analogy with [1, Lemma 4.1]. Because of this in the case $d=\pi$ we restrict our considerations to even on $x_{3}$ functions.

It follows from (6.4) and Lemma 4.1 that $\psi=\mathcal{A}_{1}(k)\left(\mathrm{I}+\mathcal{A}_{2}(k)\right)^{-1} f_{\varepsilon}$. We substitute this formula into (6.2) and obtain

$$
f_{\varepsilon}-\varepsilon \mathcal{L}_{3} \mathcal{A}_{1}(k)\left(\mathrm{I}+\mathcal{A}_{2}(k)\right)^{-1} f_{\varepsilon}=0 .
$$

By the hypothesis and Lemmas 1.4, 4.3) in the case considered the problem (1.7), (4.8) can have at most one non-trivial solution, and if exists, it satisfies (4.9). Hence, by Lemmas 5.2, 5.3, the estimate (1.3), and the definition of $\mathcal{L}_{3}$ we conclude 
that the operator $\mathcal{L}_{3} \mathcal{A}_{1}(k)\left(\mathrm{I}+\mathcal{A}_{2}(k)\right)^{-1}$ is bounded uniformly in $\varepsilon$ and small real $k$ as an operator in $L_{2}\left(\Pi_{\omega, \beta}\right)$. Thus, for $\varepsilon$ and small real $k$ the operator $\left(\mathrm{I}-\varepsilon \mathcal{L}_{3} \mathcal{A}_{1}(k)\left(\mathrm{I}+\mathcal{A}_{2}(k)\right)^{-1}\right)$ is boundedly invertible, and the equation (6.5) has the trivial solution only. Therefore, the equation (6.4) has no non-trivial solution for small $\varepsilon$ and real $k$ that completes the proof.

In the proofs of the next theorem we will employ

Lemma 6.1. Suppose that there exists a non-trivial solution $\Psi$ to (1.7), (4.9). Then

$$
\mathrm{a}(g)=-\frac{\left(\left(\mathrm{I}+\mathcal{A}_{2}(0)\right) g, \Psi\right)_{L_{2}\left(\Pi_{\omega, \beta}\right)} .}{\gamma \pi^{2}} .
$$

Proof. We denote $u:=\mathcal{A}_{1}(0) g$. This function solves (4.1), (4.8) for $k=0, f:=$ $\left(\mathrm{I}+\mathcal{A}_{2}(0)\right) g$. Now it is sufficient to integrate by parts in the integral $(f, \Psi)_{L_{2}\left(\Pi_{\omega, \beta}\right)}=$ $(f, \Psi)_{L_{2}\left(\Pi_{\omega}\right)}=-\int_{\Pi} \Psi(\Delta+1) u \mathrm{~d} x$ to prove the claimed formula.

Proof of Theorem 1.6. We argue here as in the proof of Theorem 1.5 up to the equation (6.5) $)$. We substitute the representation for $\mathcal{A}_{1}(k)\left(\mathrm{I}+\mathcal{A}_{2}(k)\right)^{-1}$ given in Lemma 5.2 into (6.5),

$$
\begin{aligned}
f_{\varepsilon} & -\varepsilon(\ln k-\ln 2+\mathrm{C}) \mathrm{a}\left(\left(\mathrm{I}+\mathcal{A}_{2}(0)\right)^{-1} f_{\varepsilon}\right) \mathcal{L}_{3} \Psi \\
& -\varepsilon \mathcal{L}_{3} \mathcal{A}_{1}(0)\left(\mathrm{I}+\mathcal{A}_{2}(0)\right)^{-1} f_{\varepsilon}-\varepsilon k^{2} \ln k \mathcal{L}_{3} \mathcal{A}_{9}(k) f_{\varepsilon}=0 .
\end{aligned}
$$

By Lemma 5.1 the operator $\mathcal{L}_{3}\left(\mathcal{A}_{1}(0)\left(\mathrm{I}+\mathcal{A}_{2}(0)\right)^{-1}+k^{2} \ln ^{3} k \mathcal{A}_{9}(k)\right)$ is bounded uniformly in $\varepsilon$ and small real $k$ as an operator in $L_{2}\left(\Pi_{\beta}\right)$. Hence, the operator

$$
\mathrm{I}-\varepsilon \mathcal{L}_{3}\left(\mathcal{A}_{1}(0)\left(\mathrm{I}+\mathcal{A}_{2}(0)\right)^{-1}+k^{2} \ln ^{3} k \mathcal{A}_{9}(k)\right)
$$

is boundedly invertible. We denote the inverse by $\mathcal{A}_{11}(\varepsilon, k)$ and apply it to (6.6),

$$
f_{\varepsilon}=\varepsilon \mathrm{a}\left(\left(\mathrm{I}+\mathcal{A}_{2}(0)\right)^{-1} f_{\varepsilon}\right) \mathcal{A}_{11}(\varepsilon, k) \mathcal{L}_{3} \Psi .
$$

We seek the non-trivial solution to (6.2). By Lemma 4.1 it implies that the associated function $f_{\varepsilon}$ is also non-trivial. Hence, by the identity obtained, a((I+ $\left.\left.\mathcal{A}_{2}(0)\right)^{-1} f_{\varepsilon}\right) \neq 0$. Taking this inequality into account, we apply the functional $\mathrm{a}\left(\left(\mathrm{I}+\mathcal{A}_{2}(0)\right)^{-1} \cdot\right)$ to (6.7) and arrive at

$$
1=\varepsilon(\ln k-\ln 2+\mathrm{C}) \mathrm{a}\left(\left(\mathrm{I}+\mathcal{A}_{2}(0)\right)^{-1} \mathcal{A}_{11}(\varepsilon, k) \mathcal{L}_{3} \Psi\right) .
$$

The roots of this equation are values of $k$ for which the equation (6.5) has a nontrivial solution. This solution is unique up to a multiplicative constant and reads as follows

$$
f_{\varepsilon}=\varepsilon(\ln k-\ln 2+\mathrm{C}) \mathcal{A}_{11}(\varepsilon, k) \mathcal{L}_{3} \Psi .
$$


The corresponding non-trivial solution to (4.1), (4.7) is given by $\psi_{\varepsilon}=\mathcal{A}_{1}(k)(\mathrm{I}+$ $\left.\mathcal{A}_{2}(k)\right)^{-1} f_{\varepsilon}$. In view of (6.8) the coefficient $\mathrm{c}$ in the asymptotics (4.7) satisfies the identity

$$
\begin{aligned}
\mathrm{c} & =-\frac{\sqrt{2 \pi}}{4 \sqrt{k}} \varepsilon \mathrm{a}\left(\left(\mathrm{I}+\mathcal{A}_{2}(0)\right)^{-1} \mathcal{A}_{11}(\varepsilon, k) \mathcal{L}_{3} \Psi\right)+\mathcal{O}(1) \\
& =\frac{\sqrt{2 \pi}}{4 \sqrt{k}(\ln k-\ln 2+\mathrm{C})}+\mathcal{O}(1), \quad k \rightarrow+0,
\end{aligned}
$$

where we have employed (6.8). Hence, $\mathrm{c}$ is non-zero and the function $\psi_{\varepsilon}$ decays at infinity (and thus belongs is a needed eigenfunction), if and only if it is associated with a positive root to (6.8). We also note that for $k=0$ the equation (6.4) can not have a non-trivial solution. Indeed, if so, it satisfies (4.8), that allows us to rewrite (6.4) as

$$
f_{\varepsilon}-\varepsilon \mathcal{L}_{3} \mathcal{A}_{1}(0)\left(\mathrm{I}+\mathcal{A}_{2}(0)\right)^{-1} f_{\varepsilon}=0 .
$$

By the boundedness of $\mathcal{A}_{11}(\varepsilon, 0)$ implies $f_{\varepsilon}=0$.

Let us study the existence of positive roots to (6.8). We rewrite the equation (6.8) as

$$
\frac{1}{\ln k-\ln 2+\mathrm{C}}-\varepsilon \mathrm{a}\left(\left(\mathrm{I}+\mathcal{A}_{2}(0)\right)^{-1} \mathcal{A}_{11}(\varepsilon, k) \mathcal{L}_{3} \Psi\right)=0 .
$$

The properties of $\mathcal{A}_{9}$ stated in Lemma 5.2 and the definition of $\mathcal{A}_{11}$ imply that the function in the left-hand side of this equation is real-valued and continuous w.r.t. small non-negative $k$. Moreover,

$$
\left|\frac{d}{d k} \mathrm{a}\left(\left(\mathrm{I}+\mathcal{A}_{2}(0)\right)^{-1} \mathcal{A}_{11}(\varepsilon, k) \mathcal{L}_{3} \Psi\right)\right| \leqslant C,
$$

where the constant $C$ is independent of $\varepsilon$ and small non-negative $k$. Hence, the derivation of the left-hand side in (6.10) is strictly negative for small positive $k$ and small $\varepsilon$. Therefore, this equation has at most one positive root. It is clear that this root exists, if

$$
\mathrm{a}\left(\left(\mathrm{I}+\mathcal{A}_{2}(0)\right)^{-1} \mathcal{A}_{11}(\varepsilon, 0) \mathcal{L}_{3} \Psi\right)<0,
$$

and does not exist, if

$$
\mathrm{a}\left(\left(\mathrm{I}+\mathcal{A}_{2}(0)\right)^{-1} \mathcal{A}_{11}(\varepsilon, 0) \mathcal{L}_{3} \Psi\right)>0 .
$$

By Lemma 6.1 and the definition of $\mathcal{A}_{11}$ we obtain that

$$
\begin{aligned}
& \mathrm{a}\left(\left(\mathrm{I}+\mathcal{A}_{2}(0)\right)^{-1} \mathcal{A}_{11}(\varepsilon, 0) \mathcal{L}_{3} \Psi\right) \\
& =\mathrm{a}\left(\left(\mathrm{I}+\mathcal{A}_{2}(0)\right)^{-1}\left(\mathrm{I}+\varepsilon \mathcal{L}_{3} \mathcal{A}_{1}(0)\left(\mathrm{I}+\mathcal{A}_{2}(0)\right)^{-1}\right) \mathcal{L}_{3} \Psi\right)+\mathcal{O}\left(\varepsilon^{2}\right) \\
& =C_{0}+\varepsilon C_{1}+\mathcal{O}\left(\varepsilon^{2}\right), \\
& C_{0}:=\frac{\left(\mathcal{L}_{4} \Psi, \Psi\right)_{L_{2}(\Pi)}}{\gamma \pi^{2}}, \quad C_{1}:=\frac{\left(\mathcal{L}_{5} \Psi, \Psi\right)_{L_{2}\left(\Pi_{\omega}\right)}+\left(\mathcal{L}_{4} \mathcal{A}_{1}(0)\left(\mathrm{I}+\mathcal{A}_{2}(0)\right)^{-1} \mathcal{L}_{4} \Psi, \Psi\right)_{L_{2}\left(\Pi_{\omega}\right)}}{\gamma \pi^{2}} .
\end{aligned}
$$


We denote $u:=\mathcal{A}_{1}(0)\left(\mathrm{I}+\mathcal{A}_{2}(0)\right)^{-1} \mathcal{L}_{4} \Psi$. It follows from Lemma 4.1 and (6.3) that $u$ is the unique solution to (4.1), (4.8) for $k=0, f=\mathcal{L}_{4} \Psi=-(\Delta+1) \beta \chi \frac{\partial \Psi}{\partial \tau}$. We denote now $\widetilde{\Psi}:=u-\beta \chi \frac{\partial \Psi}{\partial \tau}$ and conclude that there exists the unique solution to (1.7) satisfying (4.8) and (1.11). The identity (1.11) follows from the formula

$$
\Psi(x)=l_{\Psi}(s) r^{1 / 2} \sin \frac{\theta}{2}+l_{\Psi}^{(1)}(s) r \sin \theta+l_{\Psi}^{(2)}(s) r^{3 / 2} \sin \frac{3 \theta}{2}+\mathcal{O}\left(r^{2}\right), \quad r \rightarrow+0,
$$

$l_{\Psi}^{(i)} \in C^{\infty}(\partial \omega)$, which can be proved by analogy with Lemma 4.2 in [1]. Moreover, $l_{\Psi} \not \equiv 0$, since otherwise the function $\frac{\partial \Psi}{\partial x_{1}} \in \stackrel{0}{W_{2}^{1}}\left(\Pi_{\omega}\right)$ is a non-trivial solution to (1.7) belonging to $L_{2}\left(\Pi_{\omega}\right)$ that contradicts to the hypothesis. We also note that the function $u$ satisfies (1.10) with $l_{\Psi}$ replaced by $l_{\widetilde{\Psi}}$.

We employ now all the aforementioned facts and (6.3), and integrate by parts,

$$
\begin{aligned}
C_{0}= & -\frac{1}{\gamma \pi^{2}} \int_{\Pi_{\omega}} \Psi(\Delta+1) \beta \chi \frac{\partial \Psi}{\partial \tau} \mathrm{d} x=-\mathfrak{i}_{1}, \\
C_{1}= & \frac{1}{2 \gamma \pi^{2}} \int_{\Pi_{\omega}} \Psi(\Delta+1) \beta^{2} \chi^{2} \frac{\partial^{2} \Psi}{\partial \tau^{2}} \mathrm{~d} x-\frac{1}{\gamma \pi^{2}} \int_{\Pi_{\omega}} \Psi \beta \chi \frac{\partial}{\partial \tau}(\Delta+1) \beta \chi \frac{\partial \Psi}{\partial \tau} \mathrm{d} x \\
& +\frac{1}{2 \gamma \pi^{2}} \int_{\Pi_{\omega}} \Psi(\Delta+1) \frac{\left(\beta^{\prime}\right)^{2} x_{3} \chi}{2(1-\tau \mathrm{k})^{2}} \frac{\partial \Psi}{\partial x_{3}} \mathrm{~d} x+\frac{1}{\gamma \pi^{2}} \int_{\Pi_{\omega}} \Psi \beta \chi \frac{\partial}{\partial \tau}(\Delta+1) u \mathrm{~d} x \\
& -\frac{1}{\gamma \pi^{2}} \int_{\Pi_{\omega}} \Psi(\Delta+1) \beta \chi \frac{\partial u}{\partial \tau} \mathrm{d} x=-\frac{1}{\gamma \pi^{2}} \int_{\Pi_{\omega}} \Psi(\Delta+1) \beta \chi \frac{\partial u}{\partial \tau} \mathrm{d} x=-\mathfrak{i}_{2} .
\end{aligned}
$$

Hence, by (6.13),

$$
\mathrm{a}\left(\left(\mathrm{I}+\mathcal{A}_{2}(0)\right)^{-1} \mathcal{A}_{11}(\varepsilon, 0) \mathcal{L}_{3} \Psi\right)=-\mathfrak{i}_{1}-\varepsilon \mathfrak{i}_{2}+\mathcal{O}\left(\varepsilon^{2}\right) .
$$

It yields that the inequality (6.12) holds true, if the condition (1.15) is valid, i.e., in this case the operator $\mathcal{H}_{\omega_{\varepsilon}}$ has no eigenvalues converging to $1-0$ as $\varepsilon \rightarrow+0$. If the condition (1.12) is valid, it implies (6.11), and in this case the operator $\mathcal{H}_{\omega_{\varepsilon}}$ has the unique eigenvalue converging to $1-0$ as $\varepsilon \rightarrow+0$. This eigenvalues is given by $\lambda_{\varepsilon}=1-k_{\varepsilon}^{2}$, where $k_{\varepsilon}$ is the root to (6.10). The formula (6.14) and equation (6.10) yield the asymptotics for $k_{\varepsilon}$,

$$
\begin{array}{ll}
k_{\varepsilon}=2 \mathrm{e}^{-\mathrm{C}+\frac{\mathfrak{i}_{2}}{\mathfrak{i}_{1}^{2}}} \mathrm{e}^{-\frac{1}{\varepsilon i_{1}}}(1+\mathcal{O}(\varepsilon)), & \text { if } \mathfrak{i}_{1}>0, \\
k_{\varepsilon}=\mathrm{e}^{-\frac{1}{\varepsilon^{2} \mathrm{i}_{2}}}(c+\mathcal{O}(\varepsilon)), & \text { if } \mathfrak{i}_{1}=0, \quad \mathfrak{i}_{2}>0,
\end{array}
$$

where $c$ is a constant. These formulas prove (1.13).

The identities (6.8), (6.9) and the representation for $\mathcal{A}_{1}(k)\left(\mathrm{I}+\mathcal{A}_{2}(k)\right)^{-1}$ given in Lemma 5.2 imply that

$$
\psi_{\varepsilon}(x)=\Psi(x)+\mathcal{O}(\varepsilon)
$$


in $W_{2}^{1}\left(\Pi_{\omega, \widetilde{\beta}}\right)$ and $W_{2}^{2}\left(\Pi_{\omega, \widetilde{\beta}} \backslash T_{\delta}\right)$ for each $\widetilde{\beta}>0, \delta>0$. Given $\delta>0$, we can choose $\tilde{\delta}$ in (6.1) small enough so that $\widetilde{x}=x$ as $\widetilde{x} \in \Pi_{\omega, \widetilde{\beta}} \backslash T_{\widetilde{\delta}}$. Hence, by (6.15) we conclude that

$$
\psi_{\varepsilon}(x(\widetilde{x}))=\Psi(\widetilde{x})+\mathcal{O}(\varepsilon)
$$

in $W_{2}^{2}\left(S \backslash T_{\delta}\right)$ for each fixed bounded domain $S \subset \Pi_{\omega_{\varepsilon}}$ and each $\delta>0$.

We pass to the variables $\widetilde{x}$ in (6.15), and in view of last identity we conclude that the asymptotic (1.14) is valid in the norm $W_{2}^{1}\left(\Pi_{\omega, \beta}\right)$, if

$$
\left\|\varphi_{\varepsilon}\right\|_{W_{2}^{1}\left(T_{\widetilde{\delta}}\right)}=\mathcal{O}(\sqrt{\varepsilon}), \quad \varphi_{\varepsilon}=\varphi_{\varepsilon}(\widetilde{x}):=\Psi(x(\widetilde{x}))-\Psi(\widetilde{x}),
$$

for a fixed $\widetilde{\delta}>0$ small enough. Here the norm is understood in terms of variables $\widetilde{x}$. The lowest eigenvalue of Dirichlet Laplacian in $T_{\widetilde{\delta}}$ increases unboundedly as $\widetilde{\delta} \rightarrow+0$. We employ this fact, the minimax principle, and the obvious identity $\left.\varphi_{\varepsilon}\right|_{\partial T_{\widetilde{\delta}}}=0$ to conclude that for $\widetilde{\delta}$ small enough the inequality $\left\|\nabla \varphi_{\varepsilon}\right\|_{L_{2}\left(T_{\tilde{\delta}}\right)}^{2} \geqslant$ $2\left\|\varphi_{\varepsilon}\right\|_{L_{2}\left(T_{\widetilde{\delta}}\right)}^{2}$ holds true. It is also clear that the $L_{2}\left(T_{\widetilde{\delta}}\right)$-norm of $\varphi_{\varepsilon}$ is bounded uniformly in $\varepsilon$, and $\left\|\left(\Delta_{\widetilde{x}}+1\right) \varphi_{\varepsilon}\right\|_{L_{2}\left(\widetilde{T}_{\delta}\right)}=\mathcal{O}(\varepsilon)$. We employ two last relations and integrate by parts,

$$
\begin{aligned}
& \left\|\varphi_{\varepsilon}\right\|_{W_{2}^{1}\left(T_{\widetilde{\delta}}\right)}^{2} \leqslant 3\left(\left\|\nabla_{\widetilde{x}} \varphi_{\varepsilon}\right\|_{L_{2}\left(T_{\widetilde{\delta}}\right)}^{2}-\left\|\varphi_{\varepsilon}\right\|_{L_{2}\left(T_{\widetilde{\delta}}\right)}^{2}\right) \\
& =3 \int_{\partial T_{\widetilde{\delta}} \cap\left\{x: x_{3}=0\right\}} \varphi_{\varepsilon}\left[\frac{\partial \varphi_{\varepsilon}}{\partial \widetilde{x}_{3}}\right] \mathrm{d} \widetilde{x}^{\prime}-3 \int_{T_{\widetilde{\delta}}} \varphi_{\varepsilon}\left(\Delta_{\widetilde{x}}+1\right) \varphi_{\varepsilon} \mathrm{d} \widetilde{x} \\
& =3 \int_{\partial \Pi_{\omega} \backslash \partial \Pi_{\omega_{\varepsilon}}} \Psi(x(\widetilde{x}))\left[\frac{\partial \varphi_{\varepsilon}}{\partial \widetilde{x}_{3}}\right] \mathrm{d} \widetilde{x}^{\prime}-3 \int_{\partial \Pi_{\omega_{\varepsilon} \backslash \partial \Pi_{\omega}} \Psi(\widetilde{x})\left[\frac{\partial \varphi_{\varepsilon}}{\partial \widetilde{x}_{3}}\right] \mathrm{d} \widetilde{x}^{\prime}+\mathcal{O}(\varepsilon),}
\end{aligned}
$$

where

$$
\left[\frac{\partial \varphi_{\varepsilon}}{\partial \widetilde{x}_{3}}\right]:=\frac{\partial \varphi_{\varepsilon}}{\partial \widetilde{x}_{3}}\left(\widetilde{x}^{\prime},-0\right)-\frac{\partial \varphi_{\varepsilon}}{\partial \widetilde{x}_{3}}\left(\widetilde{x}^{\prime},+0\right) .
$$

It follows from (1.10) that the integrands in the remaining integrals are bounded uniformly in $\varepsilon$. Since the area of $\partial \Pi_{\omega} \backslash \partial \Pi_{\omega_{\varepsilon}}$ and $\partial \Pi_{\omega_{\varepsilon}} \backslash \partial \Pi_{\omega}$ is of order $\varepsilon$, we arrive at the identity (6.16).

The exponential decaying of $\psi_{\varepsilon}$ at infinity is due to (4.7).

\section{Acknowledgments}

I thank P. Exner, who attracted my attention to the problem studied in the paper. The part of this work was done during my visit Université de Saint-Etienne. I am grateful to the University and G. Panasenko for the hospitality extended to me.

The work is supported in parts by RFBR (06-01-00138, 05-01-97912-r_agidel). The author is also supported by Marie Curie International Fellowship within 6th European Community Framework (MIF1-CT-2005-006254). 


\section{References}

[1] D. Borisov. Discrete spectrum of a pair of non-symmetric waveguides coupled by a window // Sbornik Mathematics. 2006. V. 197. No. 4. P. 475-504.

[2] D. Borisov and P. Exner. Distant perturbation asymptotics in window-coupled waveguides. I. The non-threshold case // J. Math. Phys. 2006. V. 2006. V. 47. No. 11. P. 113502-1 - 113502-24.

[3] D. Borisov, P. Exner and R. Gadyl'shin. Geometric coupling thresholds in a two-dimensional strip // Journal of Mathematical Physics. 2002. V. 43. No. 12. P. 6265-6278.

[4] W. Bulla, F. Gesztesy, W. Renger, B. Simon. Weakly coupled bound states in quantum waveguides // Proc. Amer. Math. Soc. 1997. V. 125. No. 5. P. 1487-1495.

[5] P. Exner, P.Šeba, M. Tater, and D. Vaněk. Bound states and scattering in quantum waveguides coupled laterally through a boundary window // J. Math. Phys. 1996. V. 37. No. 10. P. 4867-4887.

[6] P. Exner and S. Vugalter. Bound-state asymptotic estimate for windowcoupled Dirichlet strips and layers // J. Phys. A. 1997. V. 30. No. 22. P. 7863-7878.

[7] R. Gadyl'shin. On local perturbations of Shrödinger operator in axis // Theor. Math. Phys. 2002. V. 132. No. 1. P. 976-982.

[8] R. Gadyl'shin. On local perturbations of Shrödinger operator on the plane // Theor. Math. Phys. 2004. V. 138. No. 1. P. 33-44.

[9] R. Gadyl'shin. On regular and singular perturbation of acoustic and quantum waveguides // Comptes Rendus Mechanique. 2004. V. 332. No. 8. P. 647-652.

[10] O.A. Ladyzhenskaya and N.N. Uraltseva. Linear and quasilinear elliptic equations, Academic Press, New York-London 1968.

[11] T. Kato. Perturbation theory for linear operators. N.Y.: Springer-Verlag, 1966.

[12] M. Klaus, and B. Simon. Coupling constants threshold in nonrelativistic quantum mechanis. I. Short-range two-body case // Ann. of Phys. 1980. V. 130. No. 2. P. 251-281.

[13] Yu. Popov. Asymptotics of bound states and bands for laterally coupled waveguides and layers // J. Math. Phys. 2002. V. 43. No. 1. P. 215-234.

[14] M. Reed, B. Simon. Methods of Modern Mathematical Physics. IV: Analysis of Operators. Academic Press, New York 1978. 